\title{
On-Line Compensation for the Disturbance Rejection Rate of a Platform Seeker Based on a High-Gain Extended State Observer
}

\author{
Li Wei $(\mathbb{D}$, Liu Shixiang $(\mathbb{D}$, Zhang Wenjie, and Xia Qunli $\mathbb{1}$ \\ School of Aerospace Engineering, Beijing Institute of Technology, Beijing 100081, China \\ Correspondence should be addressed to Li Wei; lion_lee1994@126.com
}

Received 21 February 2019; Accepted 1 July 2019; Published 8 September 2019

Academic Editor: Paolo Castaldi

Copyright ( $2019 \mathrm{Li}$ Wei et al. This is an open access article distributed under the Creative Commons Attribution License, which permits unrestricted use, distribution, and reproduction in any medium, provided the original work is properly cited.

\begin{abstract}
This paper focuses on the on-line compensation of the disturbance rejection rate (DRR) for a platform seeker. The mathematical model of the typical platform seeker based on the inertial space is established, and the line-of-sight (LOS) rate from different signal extraction points is strictly derived. Considering the spring torque disturbance and damping torque disturbance caused by the missile attitude motion, the seeker DRR transfer functions are deduced and the amplitude and phase characteristics at different frequencies are also analyzed. In order to close the engineering practice, the DRR parasitic loop (DRRPL) model of the seeker is rationally simplified and the stable region of the parasitic loop from different extraction points is also obtained. However, to increase the stability and guidance accuracy of the missile terminal flight, the compensation scheme based on the high-gain extended state observer (ESO) is used to estimate the disturbance torques and eliminate the seeker DRR effect. Numerical simulations are conducted to verify the effectiveness of the proposed scheme. The simulation results show that the seeker DRR effect mainly exists in the middle and low frequencies and the stable region of the parasitic loop at different signal extraction points is different. The proposed compensation scheme can effectively eliminate the parasitic loop effect of the seeker and increase the flight stability of the missile. It can reduce the terminal miss distance of the missile and improve the strike accuracy.
\end{abstract}

\section{Introduction}

Seekers are the tracking and detecting system of missiles, which are well used to acknowledge and track a target $[1,2]$. The accurate line-of-sight information which is extracted and then provided to the guidance and control system of the missile is aimed at making the missile fly to the target according to the predetermined guidance law. Therefore, the stability and validity of the seeker detector directly determines the terminal strike accuracy of a missile $[2,3]$. Usually, a seeker equipped with an inertial stable platform (ISP) is available to isolate the coupling effect from the attitude motion of the projectile and other external disturbances such as wind and air-stream-induced torque [4]. Actually, the seeker cannot completely eliminate the disturbance effect of body motion due to the existence of various disturbances such as wire pulling, friction, and mass imbalance, which produce various disturbance torques and reduce the tracking stability of the seeker $[5,6]$. Instead, the body motion is coupled into the output
LOS rate signals, which then induces the disturbance rejection rate characteristic of the platform seeker [6].

The DRR represents the ability of the seeker to isolate the disturbance of the missile body. It not only affects the output accuracy of the LOS rate but also forms an extra parasitic loop in the guidance system, which affects the stability and guidance accuracy $[7,8]$. At present, scholars at home and abroad pay less attention to the effect of the DRR parasitic loop on the guidance system. Cui et al. pointed out that the disturbance torque is the major factor of the DRR effect by establishing the platform seeker mathematical model [9]. Li et al. [10] analyzed the DRR model of a strapdown seeker, but the model is relatively simple, which is quite different from the DRR model of the platform seeker. Garnell [11] and Nesline and Zarchan [2] have studied the DRR induced by the radome boresight error slope and analyzed the influence on the guidance loop. Du et al. [12], Li et al. [13], and Song et al. [14] analyzed the influence of the positive feedback of DRR in a strapdown phased array seeker (SPAS) on the guidance loop stability 
and terminal guidance precision, in which the results of all these studies indicate that the positive feedback of DRR is a more severe parasitic loop.

Obviously, the DRRPL effect of the platform seeker cannot be ignored for the missile's terminal flight. Improving seeker isolation and reducing the DRR effect have also become an important research field. Two effective methods are proposed by researchers, in which one is to improve the machining accuracy and the other is to design improvements to the seeker control system $[15,16]$. However, from the perspective of the control system, estimating disturbance torque and then compensating the output information of the seeker will be a feasible method. The decoupling of the LOS rate is realized using the feedforward estimation strategy in reference [17], and this method effectively suppressed the DRR effect. Xu et al. [18] and Sadhu and Ghoshal [3] used a low-pass filter to estimate the disturbance moment and then extracted the additional LOS rate caused by the disturbance. Liu et al. [16] estimated the disturbance torque by the Kalman filtering technique to compensate for the LOS rate in the seeker output; however, the derivation process has some mistakes and the obtained results do not look very intuitive either.

As is well known, disturbance observer (DO) method has been introduced to compensate for strong nonlinearity, unmodeled dynamics, and external disturbances of uncertain systems [19-22]. Among these disturbance observers, the extended state observer, as the key module of active disturbance rejection control [23], is widely employed in various engineering control systems, such as the ESO-based finite-time tracking control for the servo system [24] and the ESO-based dynamic surface trajectory control for the quadrotor UAV [25]. Since the high-gain ESO can perform a simultaneous estimation of the state variables and the unknown disturbance [26], its interconnection in the seeker control system allows using the estimates instead of their true values to eliminate the DRR effect of the platform seeker and achieve the accurate LOS rate output.

Furthermore, Wangze [27] pointed out that the stable loop of the platform seeker has a wide bandwidth and the LOS rate output can be extracted either from the stable loop command or from the angular rate gyro. Meanwhile, his research shows that the DRR of the seeker is closed related not only to the characteristics of the control system but also to the extraction point of guidance signal and the type of disturbance torque. Based on the above foundations, a novel on-line compensation method for the DRR effect of the platform seeker is proposed in this paper. The structure of this paper is as follows. In Section 2, the mathematical model of the platform seeker considering a different guidance signal extraction point is proposed and verified. The DRR transfer functions that come up with different disturbance torque are rigorously deduced, and the corresponding amplitude and phase characteristics are analyzed in Section 2.2.1. In addition, the dynamic model of DRR is simplified equivalently, and the stable region of DRRPL is also analyzed in Section 2.2.2. In Section 3, the unknown disturbance torque is estimated, while the LOS rate output is compensated based on the high-gain
ESO technique. In Section 4, numerical simulations are conducted to verify the effectiveness of the proposed method. In Section 5, relevant conclusions to reduce the effect of DRRPL on the missile terminal guidance property are summarized.

\section{Mathematical Model of the Platform Seeker and DRR}

2.1. Extraction of the LOS Rate. The schematic diagram of the platform seeker is as depicted in Figure 1, where $o x_{b}$ $y_{b} z_{b}$ is the body coordinate and $x_{b}$ stands for axial pointing of projectile body, $x_{s}$ is the pointing seeker detector, $x_{t}$ is the orientation of the target, $\vartheta$ is the attitude angle at the pitching direction of the projectile body, $q_{s}$ is the deflection angle between the detector and the inertial space, $q_{t}$ refers to the angle of sight of the missile target, and $\varepsilon$ is the measuring error angle [15]. According to Figure 1(b), the deflection angle of stabilized platform can be expressed as

$$
\varphi(t)=q_{s}(t)-\vartheta(t)
$$

The schematic diagram of the wire disturbance is illustrated in Figure 1(a), where the stabilized platform has relative motion to the missile body in the process of real-time target detection; thus, the disturbance of the spring torque caused by the pulling of wires will hinder the movement of the frame [28]. The disturbance of the spring torque can be expressed as

$$
T_{s}(t)=K_{s} \varphi(t) .
$$

Another major disturbance named as the damping torque is related to the rotation angular rate of the seeker platform, which can be expressed as

$$
T_{v}(t)=K_{w}\left(\dot{q}_{s}(t)-\dot{\vartheta}(t)\right)=K_{w} \dot{\varphi}(t)
$$

In Equations (2) and (3), $\dot{\varphi}$ is the deflection angular rate of the stabilized platform and $K_{s}$ and $K_{w}$ are the spring torque coefficient and damping torque coefficient, respectively. Then, the motor dynamic model with the disturbance torque can be obtained as

$$
J \dot{q}_{s}=T_{M}-K_{w} \dot{\varphi}-K_{s} \varphi,
$$

where $J$ is the rotational inertia of the platform and $T_{M}$ is the output torque of servo motor. However, to simplify the seeker model, the disturbance of the spring torque and the damping torque can be uniformly represented by $G_{D}(s)$, which expressed as

$$
G_{D}(s)=K_{w}+\frac{K_{s}}{s}
$$

To form a closed loop for the stable platform, the LOS rate in the inertia space feedback is acquired via the rate 




(a) Wire disturbance



(b) The angle relationship of the pitch plane

FIGURE 1: The structure of the platform seeker.

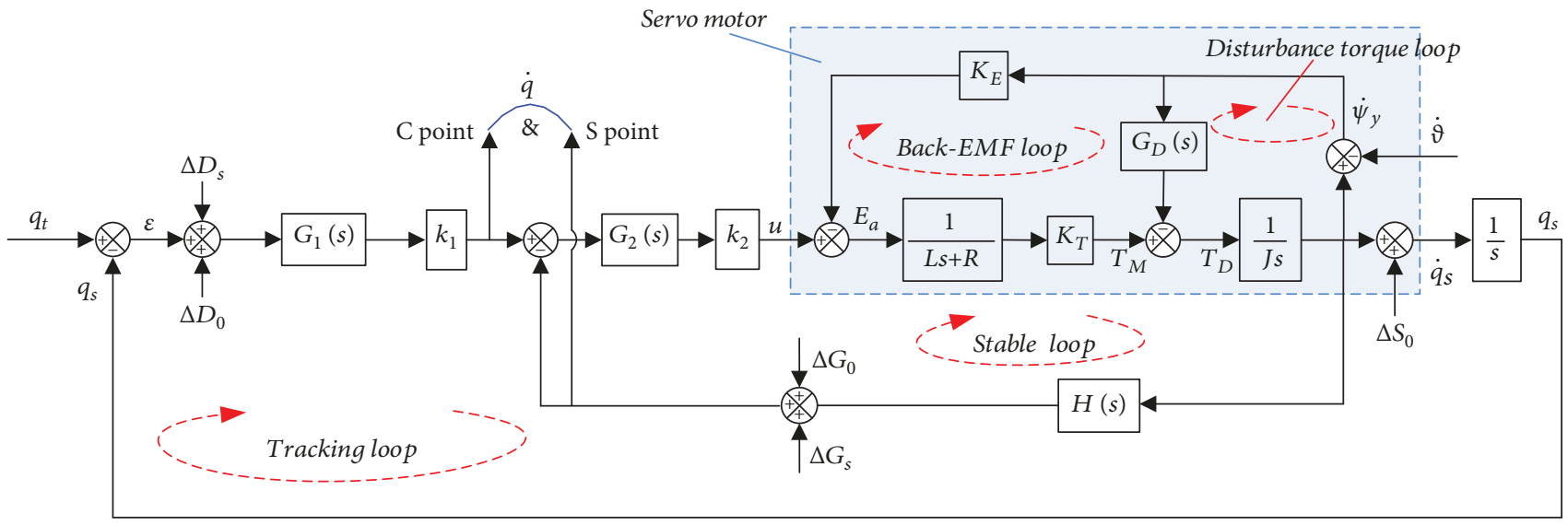

FIGURE 2: Schematic diagram of the platform seeker tracking control system.

gyro. The schematic diagram of a typical platform seeker tracking control system is shown in Figure $2[29,30]$, where $G_{1}(s)$ and $G_{2}(s)$ are the transfer functions of the correction network for the tracking loop and stable loop, respectively. $k_{1}$ and $k_{2}$ are the forward gains of the operational amplifier. In addition, $L$ and $R$, respectively, indicate the inductance and capacitance of the servo machine winding, and $K_{T}$ is the moment coefficient of the servo motor mounted on a platform frame, and $K_{E}$ is the back electromotive force coefficient. $G_{D}(s)$ is the transfer function of the disturbance torque, while $H(s)$ is the transfer function of the angular rate gyro.

As shown in Figure 2, the tracking control system is used to achieve accurate tracking to the theoretical LOS angle, which mainly includes a stable loop, tracking loop, backEMF loop, and disturbance torque loop. The disturbance torque loop couples the base motion to the seeker and reduces the precision. The back-EMF loop is generally small, and thus, it minimally affects seeker control precision. The LOS rate signals transferred to the missile guidance system can be obtained from the $\mathrm{C}$ point and the $\mathrm{S}$ point. Thus, the transfer functions of the platform seeker by different signal points can be expressed as

$$
\begin{aligned}
G_{\dot{q}}^{C}(s) & =\frac{\dot{q}_{s}^{C}(s)}{\dot{q}_{t}(s)} \\
& =\frac{G_{1}(s) k_{1}\left[(L s+R) J s+G_{D}(s)(L s+R)+G_{2}(s) H(s) k_{2} K_{T}\right]}{s\left[J(L s+R)+G_{D}(s)(L s+R)+G_{2}(s) H(s) k_{2} K_{T}\right]+G_{1}(s) k_{1} G_{2}(s) k_{2} K_{T}},
\end{aligned}
$$

$$
\begin{aligned}
G_{\dot{q}}^{S}(s) & =\frac{\dot{q}_{s}^{S}(s)}{\dot{q}_{t}(s)} \\
& =\frac{G_{1}(s) G_{2}(s) H(s) k_{1} k_{2} K_{T}}{s\left[J(L s+R)+G_{D}(s)(L s+R)+G_{2}(s) H(s) k_{2} K_{T}\right]+G_{1}(s) k_{1} G_{2}(s) k_{2} K_{T}} .
\end{aligned}
$$

High-frequency dynamics, correction networks, and small values can be neglected in engineering [14-16]. Assuming $G_{1}(s)=1, G_{2}(s)=1, R \approx 1$, and $L \approx 0$, the equivalent stable loop gain is $K_{2}=k_{2} K_{T} G_{2}(s) / J$ and the equivalent 
tracking loop gain is $K_{1}=k_{1} G_{1}(s)$. Then Equations (6) and (7) can be simplified as

$$
\begin{aligned}
& G_{\dot{q}}^{C}(s)=\frac{\dot{q}_{s}^{C}(s)}{\dot{q}_{t}(s)}=\frac{K_{1}\left[J s+G_{D}(s)+K_{2}\right]}{J s^{2}+\left[G_{D}(s)+K_{2}\right] s+K_{1} K_{2}}, \\
& G_{\dot{q}}^{S}(s)=\frac{\dot{q}_{s}^{S}(s)}{\dot{q}_{t}(s)}=\frac{K_{1} K_{2}}{J s^{2}+\left[G_{D}(s)+K_{2}\right] s+K_{1} K_{2}} .
\end{aligned}
$$

In fact, for a perfect seeker, the influence of disturbance $G_{D}(s)$ on the tracking characteristic is usually neglected when it is relatively small [15], and then, Equations (8) and (9) can both be transformed into

$$
G_{\dot{q}}^{C}(s) \approx G_{\dot{q}}^{S}(s) \approx \frac{\dot{q}_{s}}{\dot{q}_{t}}=\frac{1}{s / K_{1}+1},
$$

It is obvious from Equation (10) that the seeker dynamics of the LOS rate signal responses from the $\mathrm{C}$ point and the $S$ point are consistent when neglecting the effects of zero deviations and detector noises on the control system of the seeker.

The typical parameters of a platform seeker are shown as in Table 1, and the step responses of the seeker tracking control system without considering any disturbances are shown in Figure 3(a). But for an imperfect seeker, the related zero deviations and detector noises are introduced, and for this purpose, it is assumed that the variances of the seeker detector noise $\left(\Delta D_{s}\right)$ and rate gyro noise $\left(\Delta G_{s}\right)$ are $0.0001 \% \mathrm{~s}^{2}$ and $0.001 \% \mathrm{~s}^{2}$, respectively. The zero deviations of the seeker detector $\left(\Delta D_{0}\right)$ and servo system $\left(\Delta S_{0}\right)$ are both chosen as $0.1 \% \mathrm{~s}^{2}$. Then, the simulation results under various disturbances are shown in Figure 3(b)-3(f).

It can be observed from Figure 3(a) that the output of the LOS rate from the $\mathrm{S}$ point lags slightly behind the $\mathrm{C}$ point, while remaining consistent basically in Figure $3(\mathrm{~b})-3(\mathrm{~d})$. However, in Figure 3(b)-3(d), the steady-state gains of the output responses exist at a certain deviation compared with the expected LOS rate. In Figure 3(e), the influence of the seeker detector noise on the signal responses from the $\mathrm{C}$ point is larger than the $\mathrm{S}$ point because of the filtering effect of the seeker control system, while the opposite conclusion can be obtained in Figure 3(f). Finally, compared with Figures 3(e) and 3(f), it can be seen that the detector noise has a greater impact on the seeker control system than on the rate gyro noise.

\subsection{Analysis of $D R R P L$}

2.2.1. The DRR Model of the Platform Seeker. The DRR represents the capability of the isolation base motion of a missile, which can guarantee a high-precision LOS rate. The smaller the DRR is, the stronger the ability of the isolation base disturbance the seeker has. Thus, the DRR transfer function in Figure 2 can be expressed as

$$
R(s)=\frac{\Delta \dot{q}(s)}{\dot{\vartheta}(s)},
$$

TABLE 1: Typical parameters of a platform seeker.

\begin{tabular}{lc}
\hline Parameter & Value \\
\hline Tracking loop gain & $k_{1}=12$ \\
Stable loop gain & $k_{2}=12$ \\
Inductance & $L=0.0035 \mathrm{H}$ \\
Resistance & $R=8 \Omega$ \\
Moment coefficient of servo motor & $K_{T}=0.2334 \mathrm{~N} \cdot \mathrm{m} / \mathrm{A}$ \\
Rotational inertia & $J=0.0020995 \mathrm{~kg} \cdot \mathrm{m}^{2}$ \\
Back-EMF coefficient & $K_{E}=0.2344 \mathrm{~V} / \mathrm{rad} / \mathrm{A}$ \\
\hline
\end{tabular}

where $\dot{\vartheta}(s)$ is the attitude angular rate of the missile and $\Delta \dot{q}(s)$ is the coupled LOS rate of the seeker induced by the missile motion. According to the different signal extraction methods shown in Figure 2, ignoring the influence of the back-EMF loop and angular rate gyro dynamic $H(s)$, the DRR transfer functions of the seeker are obtained as

$$
\begin{aligned}
R(s)^{C} & =\frac{\Delta \dot{q}^{C}(s)}{\dot{\vartheta}(s)} \\
& =\frac{-\left[G_{1}(s) G_{D}(s) k_{1}(L s+R)\right]}{s\left[J s(L s+R)+G_{D}(s)(L s+R)+G_{2}(s) k_{2} K_{T}\right]+G_{1}(s) G_{2}(s) k_{1} k_{2} K_{T}},
\end{aligned}
$$

$$
\begin{aligned}
R(s)^{S} & =\frac{\Delta \dot{q}^{S}(s)}{\dot{\vartheta}(s)} \\
& =\frac{s\left[G_{D}(s)(L s+R)\right]}{s\left[J s(L s+R)+G_{D}(s)(L s+R)+G_{2}(s) k_{2} K_{T}\right]+G_{1}(s) G_{2}(s) k_{1} k_{2} K_{T}} .
\end{aligned}
$$

The DRR amplitude ratio of the $\mathrm{C}$ point to the $\mathrm{S}$ point is $-k_{1} / s$, which can be obtained by dividing Equation (12) by Equation (13) regardless of the correction link $G_{1}(s)$. Thus, no matter what the disturbance torque is, the relation between DRR functions from the $\mathrm{C}$ point and the $\mathrm{S}$ point is inversely proportional to the operating frequency of the seeker and proportional to the forward gain $k_{1}$ of the tracking loop, as shown in Figure 4.

By substituting Equations (2) and (3) into Equation (12), the DRR transfer functions from the $\mathrm{C}$ point can be obtained, as shown in

$$
\begin{aligned}
R(s)_{v}^{C} & =\frac{\Delta \dot{q}_{v}^{C}(s)}{\dot{\vartheta}(s)} \\
& =\frac{-\left[K_{w} k_{1}(L s+R) G_{1}(s)\right]}{s\left[J s(L s+R)+K_{w}(L s+R)+k_{2} K_{T} G_{2}(s)\right]+k_{1} k_{2} K_{T} G_{1}(s) G_{2}(s)},
\end{aligned}
$$

$$
\begin{aligned}
R(s)_{s}^{C} & =\frac{\Delta \dot{q}_{s}^{C}(s)}{\dot{\vartheta}(s)} \\
& =\frac{-\left[K_{s} k_{1} G_{1}(s)(L s+R)\right]}{s^{2}\left[J s(L s+R)+k_{2} K_{T} G_{2}(s)\right]+\left[K_{s}(L s+R)+k_{1} k_{2} K_{T} G_{1}(s) G_{2}(s)\right] s} .
\end{aligned}
$$




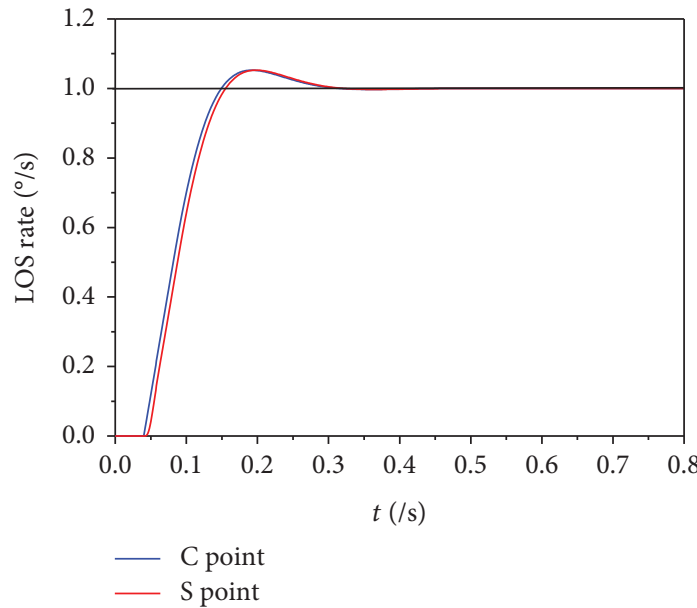

(a) Without any disturbance



- C point

- S point

(c) Zero deviation of rate gyro

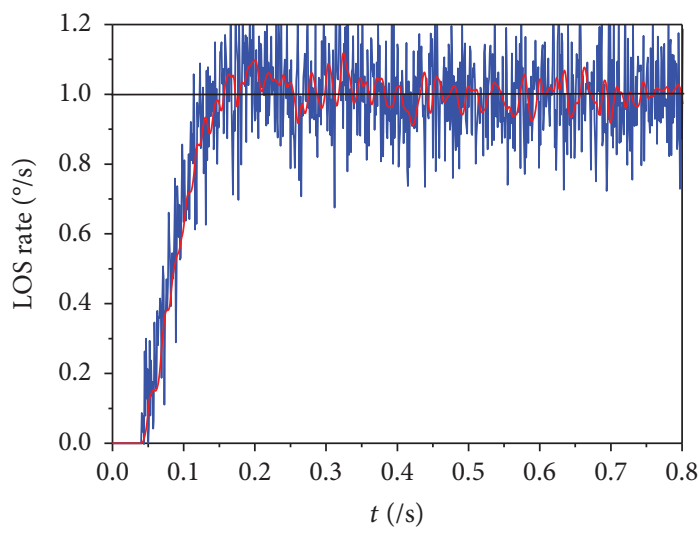

point

$S$ point

(e) Detector noise

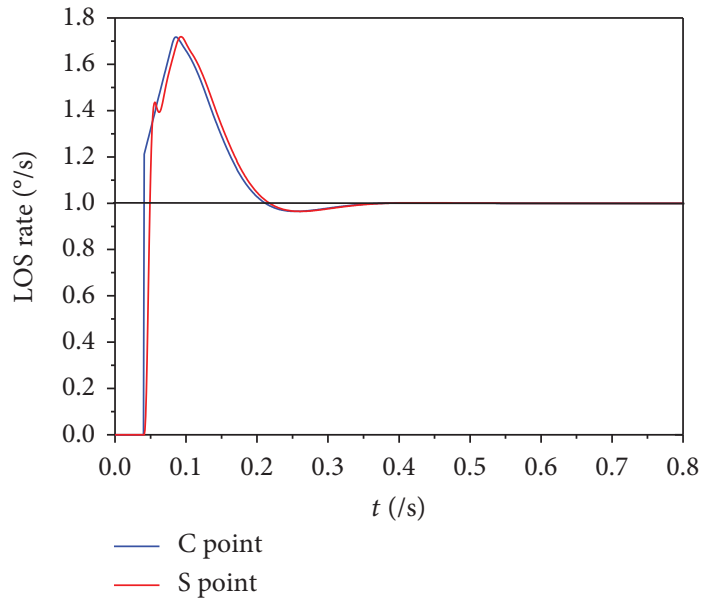

(b) Zero deviation of detector

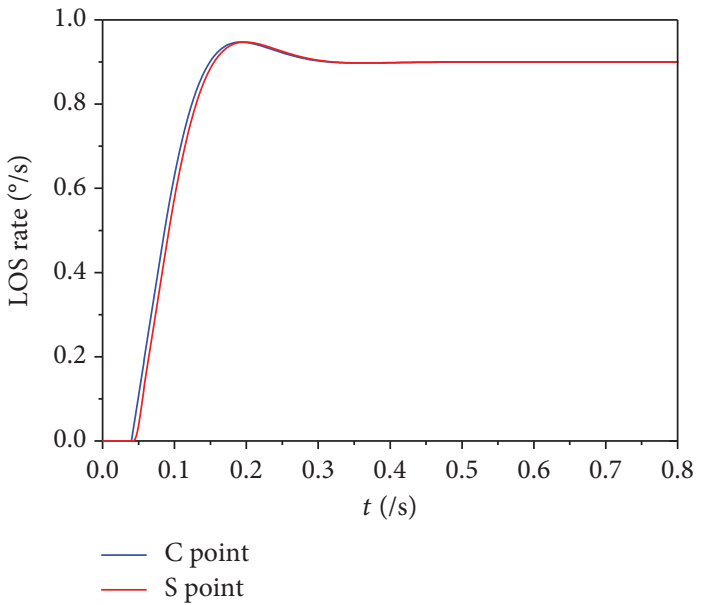

(d) Zero deviation of servo system

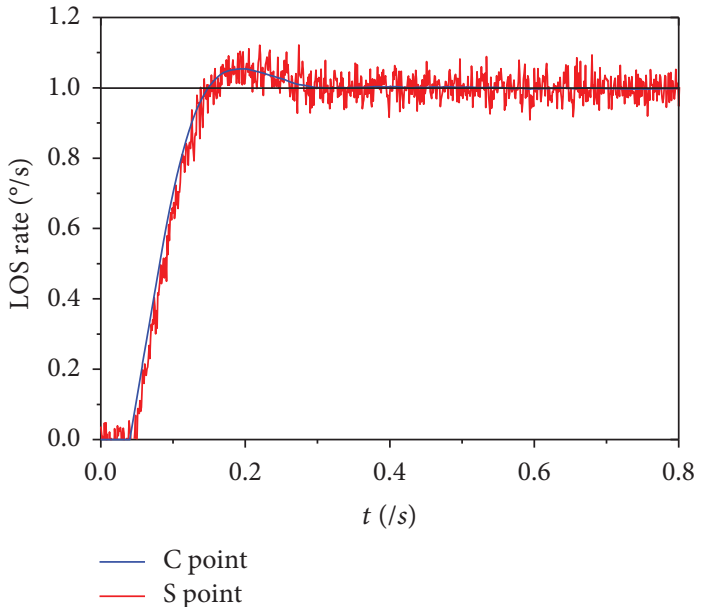

(f) Rate gyro noise

FIgURE 3: LOS rate extraction from the $\mathrm{C}$ point and $\mathrm{S}$ point. 


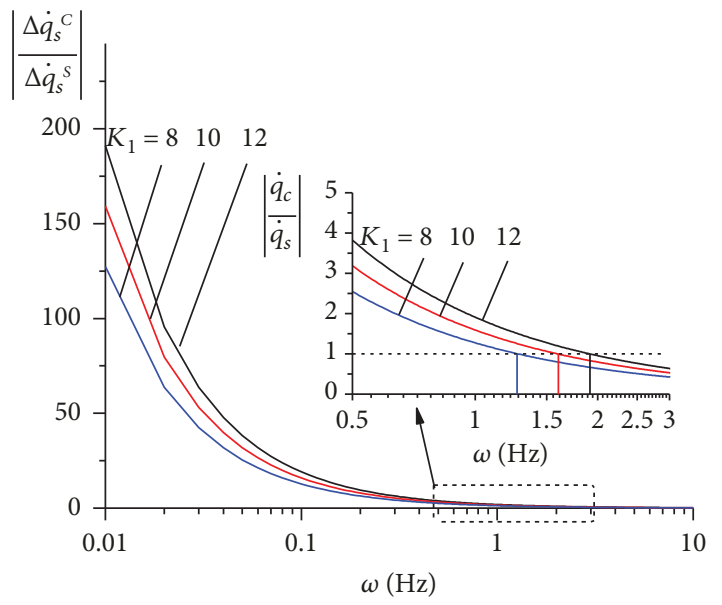

(a) Proportional coordinate

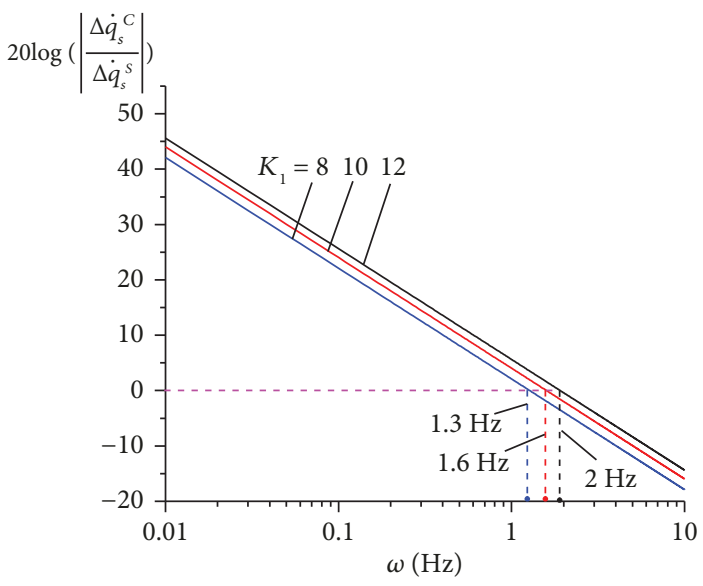

(b) Logarithmic coordinate

FIgURE 4: The DRR amplitude ratio of the $\mathrm{C}$ point to the $\mathrm{S}$ point.

Similarly, the DRR transfer functions from the $\mathrm{S}$ point can also be obtained as follows:

$$
\begin{aligned}
R(s)_{v}^{S} & =\frac{\Delta \dot{q}_{v}^{S}(s)}{\dot{\vartheta}(s)} \\
& =\frac{s\left[K_{w}(L s+R)\right]}{s\left[J s(L s+R)+K_{w}(L s+R)+G_{2}(s) k_{2} K_{T}\right]+G_{1}(s) G_{2}(s) k_{1} k_{2} K_{T}},
\end{aligned}
$$

$$
\begin{aligned}
R(s)_{s}^{S} & =\frac{\Delta \dot{q}_{s}^{S}(s)}{\dot{\vartheta}(s)} \\
& =\frac{K_{s}(L s+R)}{s\left[J s(L s+R)+k_{2} K_{T} G_{2}(s)\right]+k_{1} k_{2} K_{T} G_{1}(s) G_{2}(s)+K_{s}(L s+R)} .
\end{aligned}
$$

From Equations (14)-(17), it is obvious that the DRR transfer functions of the seeker are different for different disturbance torques and guidance signal extraction points. Therefore, the frequency characteristic of the seeker DRR is closely related to the frequency characteristic of the seeker control system and cannot be simply regarded as a constant value of a certain frequency point.

Based on Equations (14)-(17), and the typical parameters of a platform seeker which are given in Table 1, the DRR transfer function Bode diagrams of different disturbance torques and guidance signal extraction points are shown in Figure 5.

According to Figure 5, the amplitude frequency characteristic of the seeker DRR attenuates seriously in the highfrequency band, so it can be inferred that the influence of DRR on the performance of the seeker control system mainly exists in the middle and low frequency band. From the phase frequency characteristic, the initial phase of the DRR transfer function from the $S$ point under the spring torque and damping torque is $0^{\circ}$ and $90^{\circ}$, whereas the initial phase from the $\mathrm{C}$ point is $-270^{\circ}$ and $-180^{\circ}$.
2.2.2. Stable Region Analysis of DRRPL. In engineering, the seeker DRR model established in Section 2.2.1 can be simplified under the following assumptions $[15,16,31]$ :

(a) High-frequency dynamics, correction links, and small values can be neglected in the seeker DRR analysis, i.e., $G_{1}(s)=1, G_{2}(s)=1, R \approx 1$, and $L \approx 0$

(b) The back-EMF loop is generally small, and thus, it minimally affects seeker control precision

(c) Ignoring the rate gyro dynamics and the feedback gain of the stable loop is equal to 1 (i.e., $k_{g}=1$ )

(d) The forward gain of the stable loop is equivalent to $K_{2}=k_{2} K_{T} G_{2}(s) / J$, and the forward gain of the tracking loop is equivalent to $K_{1}=k_{1} G_{1}(s)$

Based on the above assumptions, the schematic diagram of platform seeker shown in Figure 2 can be transformed into Figure 6 , where $\ddot{q}_{D}$ is the disturbance angular acceleration induced by disturbance torques.

Defining $K_{s}^{\prime}=K_{s} / J$ and $K_{w}^{\prime}=K_{w} / J$, Equations (14) and (15) can then be rewritten as Equations (18) and (19), respectively:

$$
R(s)_{v}^{C}=\frac{\Delta \dot{q}_{v}^{C}(s)}{\dot{\vartheta}(s)}=\frac{-K_{w}^{\prime} / K_{2}}{\left(1 / K_{1} K_{2}\right) s^{2}+\left(\left(K_{w}^{\prime}+K_{2}\right) / K_{1} K_{2}\right) s+1},
$$

$$
\begin{aligned}
R(s)_{s}^{C} & =\frac{\Delta \dot{q}_{s}^{C}(s)}{\dot{\vartheta}(s)} \\
& =\frac{-K_{s}^{\prime} K_{1} /\left(K_{s}^{\prime}+K_{1} K_{2}\right)}{s\left[\left(1 /\left(K_{s}^{\prime}+K_{1} K_{2}\right)\right) s^{2}+\left(K_{2} /\left(K_{s}^{\prime}+K_{1} K_{2}\right)\right) s+1\right]} .
\end{aligned}
$$




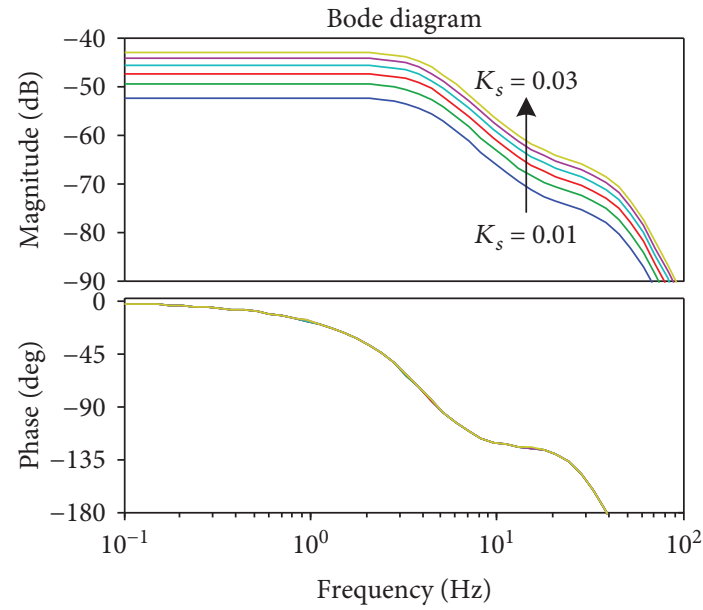

(a) S point under spring torque

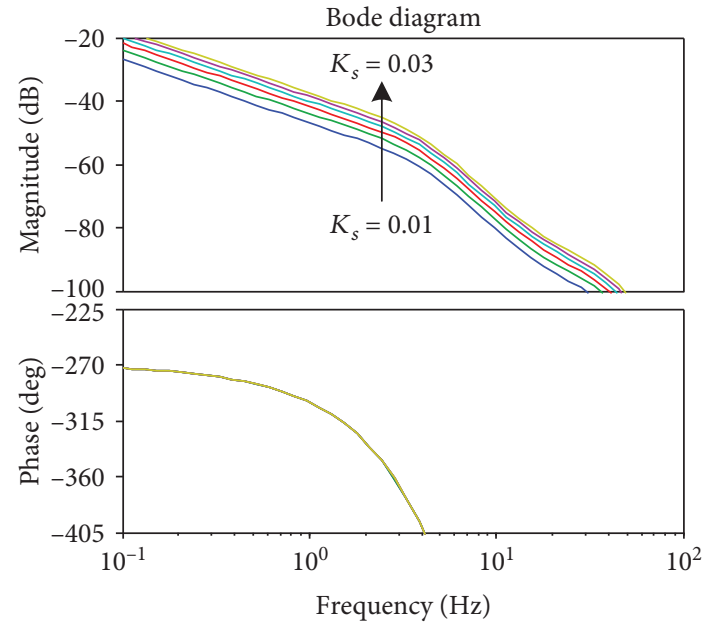

(c) C point under spring torque

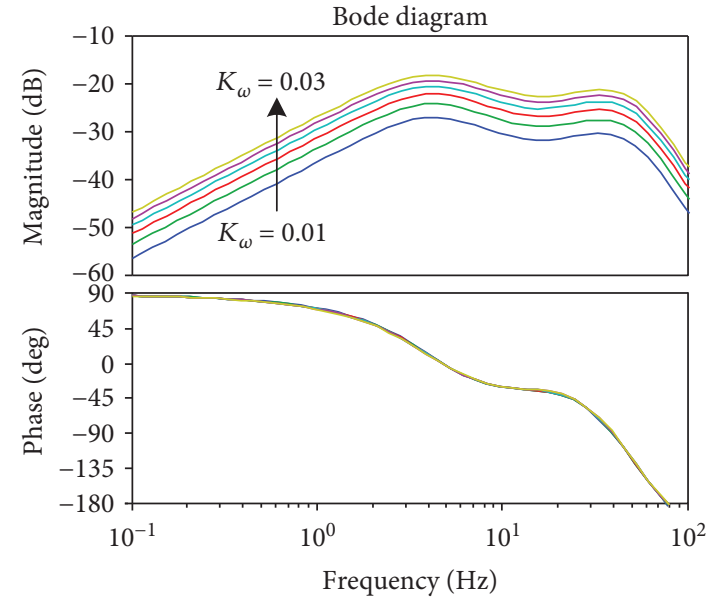

(b) $\mathrm{S}$ point under damping torque

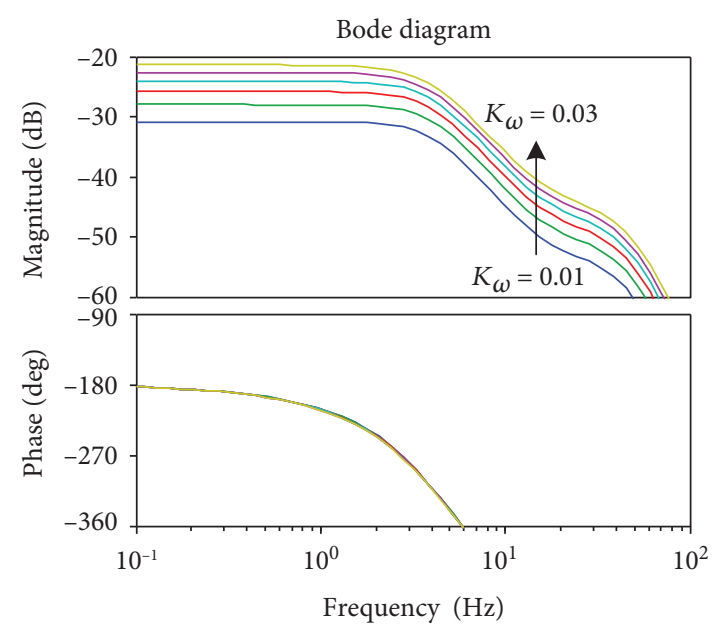

(d) C point under damping torque

Figure 5: Bode diagrams of the DRR characteristic.



FIgURE 6: Equivalent block diagram of the DRR. 
TABLE 2: First-order DRR transfer function of the seeker.

\begin{tabular}{lccc}
\hline & \multicolumn{1}{c}{ C point } & S point & Equivalent coefficient \\
\hline Damping torque & $R(s)_{v}^{C}=-R_{w} / T_{d} s+1$ & $R(s)_{v}^{S}=R_{w} s / K_{1} / T_{d} s+1$ & $R_{w}=K_{w}^{\prime} / K_{2}, T_{d}=K_{w}^{\prime}+K_{2} / K_{1} K_{2}$ \\
Spring torque & $R(s)_{s}^{C}=-R_{s} /\left(T_{s} s+1\right) s$ & $R(s)_{s}^{S}=R_{s} / K_{1} / T_{s} s+1$ & $R_{s} \approx K_{s}^{\prime} / K_{2}, T_{s}=K_{2} / K_{1} K_{2}+K_{s}^{\prime}$ \\
\hline
\end{tabular}

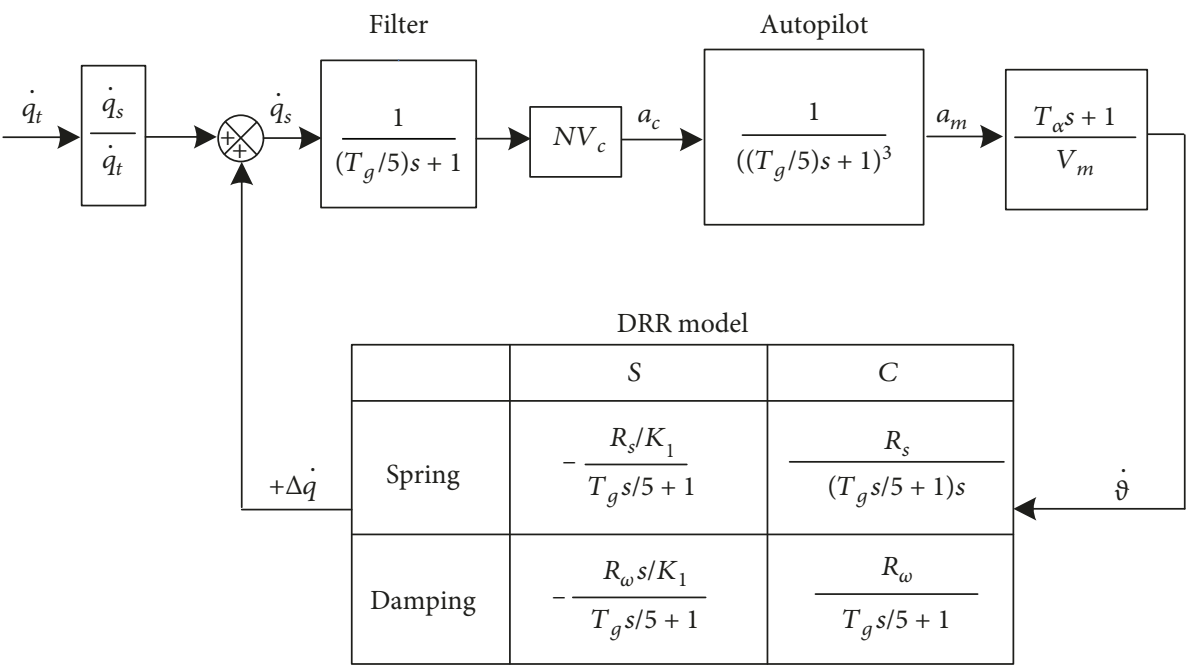

FIgURE 7: The simple structure of the DRR parasitic loop.

Similarly, Equations (16) and (17) can be rewritten as Equations (20) and (21), respectively.

$$
\begin{aligned}
R(s)_{v}^{S} & =\frac{\Delta \dot{q}_{v}^{S}(s)}{\dot{\vartheta}(s)}=\frac{K_{w}^{\prime} s / K_{1} K_{2}}{\left(1 / K_{1} K_{2}\right) s^{2}+\left(\left(K_{w}^{\prime}+K_{2}\right) / K_{1} K_{2}\right) s+1}, \\
R(s)_{s}^{S} & =\frac{\Delta \dot{q}_{s}^{S}(s)}{\dot{\vartheta}(s)} \\
& =\frac{K_{s}^{\prime} /\left(K_{s}^{\prime}+K_{1} K_{2}\right)}{\left(1 /\left(K_{s}^{\prime}+K_{1} K_{2}\right)\right) s^{2}+\left(K_{2} /\left(K_{s}^{\prime}+K_{1} K_{2}\right)\right) s+1} .
\end{aligned}
$$

According to Table 1 , there exists $K_{1} K_{2} \gg 1$ and $K_{1}$ $K_{2} \gg K_{s}^{\prime}$ and the DRR transfer functions shown in Equations (18)-(21) can be simplified to a first-order dynamic model, as shown in Table 2.

In the terminal guidance phase, the seeker provides the guidance signal for the missile. However, if the seeker cannot isolate the body disturbance perfectly, the guidance signal not only includes the real LOS rate but also includes the additional signal $\Delta \dot{q}$ which is caused by missile attitude motion. Then, the parasitic loop is stimulated, which is called the DRRPL. In order to analyze the characteristic of DRRPL intuitively, the simple structure of the DRR parasitic loop is shown in Figure 7. The guidance system is regarded as a five-order system with guidance time constant $T_{g}$ [30], in which the guidance filter can be simplified as $G_{F}(s)=$ $1 /\left(T_{g} s / 5+1\right)$, whereas the autopilot is the third-order model $G_{a}(s)=1 /\left(T_{g} s / 5+1\right)^{3}$. The seeker is regarded as the first-order model with $T_{\text {seeker }}=T_{g} / 5 . T_{\alpha}$ is the time constant of the angle of attack, which represents the rapidity of missile attitude response.

$$
\begin{aligned}
G(s) & =\frac{a_{m}}{\dot{q}_{t}} \\
& =\frac{N V_{c}}{\left(T_{g} s / 5+1\right)^{5}-N\left(V_{c} / V_{m}\right)\left(T_{\alpha} s+1\right)\left(T_{g} s / 5+1\right) R(s)} .
\end{aligned}
$$

According to Figure 7, the closed-loop transfer function of the DRR parasitic loop can be obtained, as shown in Equation (22), where $R(s)$ is the transfer function of the DRR model. Furthermore, the stable region of the DRRPL can be obtained, as shown in Figure 8 .

It can be observed from Figure 8 that the stable region of the DRRPL decreases with the increase of the disturbance torque coefficients $R_{w}$ and $R_{s}$. The stable region under the damping torque from the $C$ point is wider than that from the $S$ point, whereas the stable region under the spring torque from the $C$ point is less than that from the $S$ point. Thus, the stable region of DRRPL should be analyzed considering the main disturbance torques of the seeker caused by the trajectory characteristics, so as to select the appropriate extraction point of the LOS rate during the terminal-guided flight. 


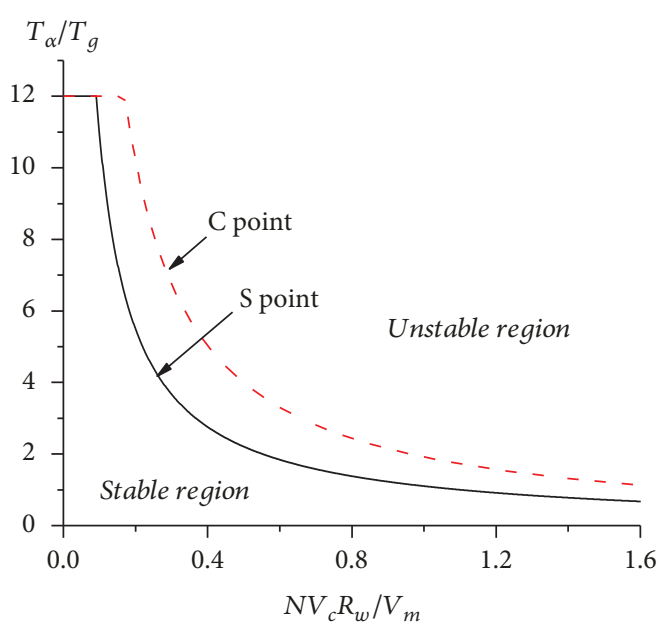

(a) Damping torque

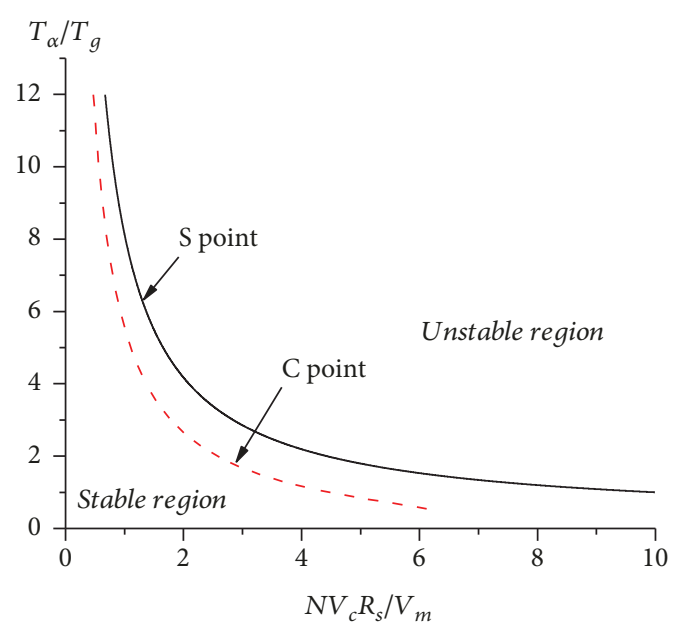

(b) Spring torque

Figure 8: Stable region of the DRRPL.

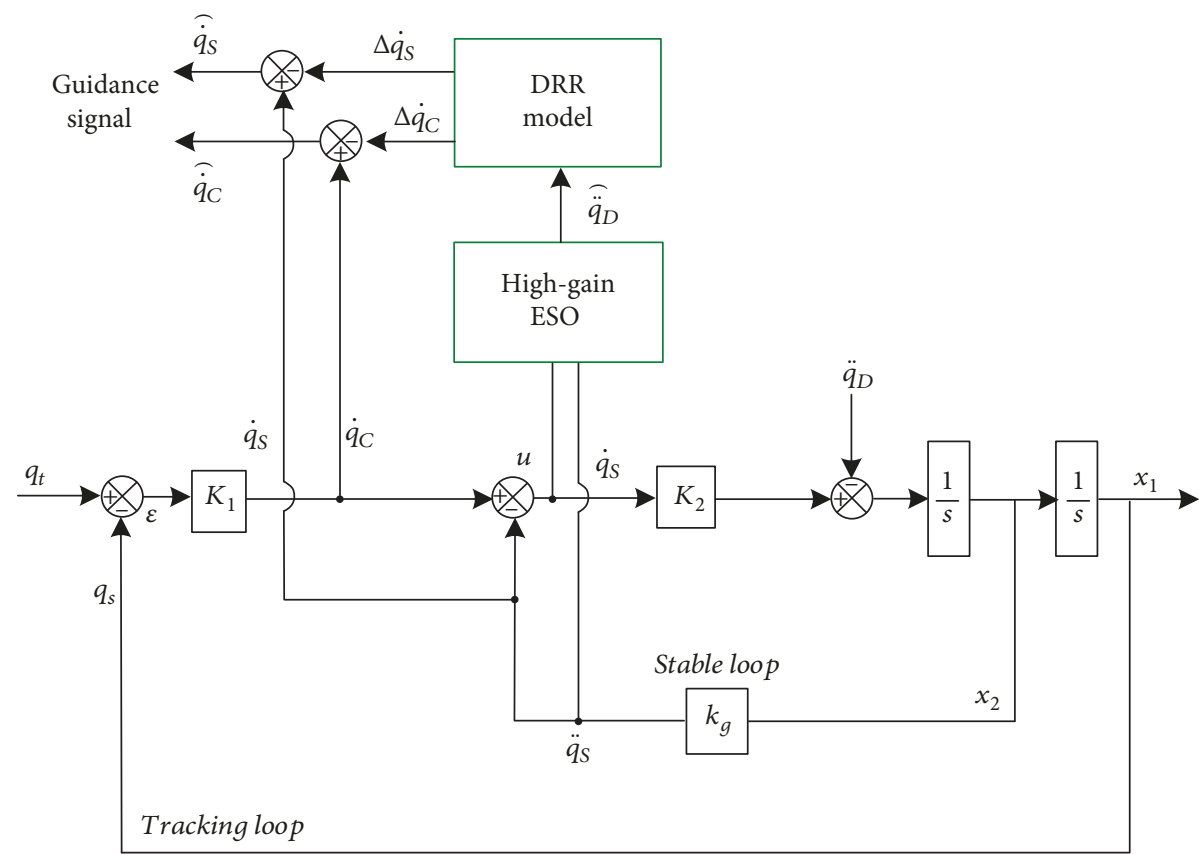

FIgURe 9: Compensation scheme of DRR.

\section{DRR Estimation and Compensation}

Based on the analysis in Section 2, it is known that the existence of the seeker DRR will inevitably affect the terminal strike accuracy of the missile, and the DRR effect is time-varying because the disturbance induced by the body attitude motion is uncertain. Secondly, the stable region of the DRRPL will decrease with the increase of the disturbance torque. The above conclusions indicate that it is necessary for the seeker to compensate for the DRR effect in real time. Therefore, in this section, the additional LOS rate caused by the disturbance torque is quickly estimated based on the ESO to compensate for the output of the seeker.
3.1. ESO-Based Estimation of Disturbance Torque. The specific model of the seeker disturbance torque is ignored, and the influence of the disturbance torque on the tracking control system of the seeker is replaced by the interference angular acceleration $\ddot{q}_{D}$, as shown in Figure 9. Consistent with Section 2.2.2, the seeker stable loop gain and tracking loop gain are represented by $K_{2}$ and $K_{1}$, respectively. The high-gain ESO is used to estimate the angular acceleration, and then the additional LOS rate signals $\Delta \dot{q}_{c}$ and $\Delta \dot{q}_{s}$ are calculated according to the DRR model of the platform seeker. Finally, the compensated guidance signals $\widehat{\dot{q}}_{c}$ and $\widehat{\dot{q}}_{s}$ can be transmitted to the autopilot to achieve the flight control of the missile. 
Based on Figure 9, the system state equations of the platform seeker can be established as

$$
\left\{\begin{array}{l}
\dot{x}_{1}=x_{2} \\
\dot{x}_{2}=K_{2} u-\ddot{q}_{D} \\
y=x_{1}
\end{array}\right.
$$

where $x_{1}$ and $x_{2}$ are the state variables, which represent $q_{s}$ and $\dot{q}_{s}$, respectively. $u$ is the control input of the servo motor. Defining an extended state, $x_{3} \triangleq\left(-\ddot{q}_{D}\right)$ for Equation (23), and the new extended order system can be written as

$$
\left\{\begin{array}{l}
\dot{x}_{1}=x_{2} \\
\dot{x}_{2}=x_{3}+K_{2} u \\
\dot{x}_{3}=w(t) \\
y=x_{1}
\end{array},\right.
$$

where $w(t)$ is the rate of change of the disturbance angular acceleration, $\ddot{q}_{D}$, and is assumed to be an unknown but bounded quantity. By making $\ddot{q}_{D}$ a state, however, it is now possible to estimate it by using the state estimator. To this end, consider a nonlinear observer of the form $[32-34]$

$$
\left\{\begin{array}{l}
e_{1}=z_{1}-y \\
\dot{z}_{1}=z_{2}-\frac{\beta_{01}}{\varepsilon}\left|e_{1}\right|^{\alpha_{1}} \operatorname{sign}\left(e_{1}\right) \\
\dot{z}_{2}=z_{3}-\frac{\beta_{02}}{\varepsilon^{2}}\left|e_{1}\right|^{\alpha_{2}} \operatorname{sign}\left(e_{1}\right)+K_{2} u \\
\dot{z}_{3}=-\frac{\beta_{03}}{\varepsilon^{3}}\left|e_{1}\right|^{\alpha_{3}} \operatorname{sign}\left(e_{1}\right)
\end{array}\right.
$$

where $\beta_{01}>0, \beta_{03}>0$, and $\beta_{02}>\beta_{03} / \beta_{01}$ and $\alpha_{3}=\alpha \in(0,1)$, $\alpha_{2}=\left(2 \alpha_{3}+1\right) / 3, \alpha_{1}=\left(\alpha_{3}+2\right) / 3$, and $\varepsilon \in(0,1) . z_{1}, z_{2}$ and $z_{3}$ stand for the estimate values of $q_{s}, \dot{q}_{s}$ and $-\ddot{q}_{D}$, respectively.

Theorem 1. Supposing $e_{1}=z_{1}-y, e_{2}=z_{2}-\dot{y}$, and $e_{3}=z_{3}+$ $\ddot{q}_{D}$ stand for the estimation errors. Considering the extended system (24) and observer system (25), and that $L>0, \sigma=(1-\gamma) / \gamma$ exists, $\gamma \in(0,1 / 2)$ makes the estimation errors stabilized in the following internals with finite time, as shown:

$$
\begin{gathered}
\left|z_{1}-y\right| \leq L \varepsilon^{3 \sigma} \\
\left|z_{2}-\dot{y}\right| \leq L \varepsilon^{3 \sigma-1} \\
\left|z_{3}-x_{3}\right| \leq L \varepsilon^{3 \sigma-2}
\end{gathered}
$$

Proof. The error equations between the extended system (see Equation (24)) and the observer system (see Equation (25)) can be obtained as

$$
\left\{\begin{array}{l}
\dot{e}_{1}=e_{2}-\frac{\beta_{01}\left|e_{1}\right|^{\alpha_{1}} \operatorname{sign}\left(e_{1}\right)}{\varepsilon} \\
\dot{e}_{2}=e_{3}-\frac{\beta_{02}\left|e_{1}\right|^{\alpha_{2}}}{\varepsilon^{2}} \operatorname{sign}\left(e_{1}\right) \\
\dot{e}_{3}=-\frac{\beta_{03}\left|e_{1}\right|^{\alpha_{3}}}{\varepsilon^{3}} \operatorname{sign}\left(e_{1}\right)-w(t)
\end{array} .\right.
$$

Then, considering the following coordinate transformation

$$
\begin{aligned}
\tau & =\frac{t}{\varepsilon}, \\
\eta(t) & =\varepsilon^{3} w(t), \\
w_{i}(\tau) & =\varepsilon^{i-1} e_{i}(t), \quad i=1,2,3 .
\end{aligned}
$$

Thus, Equation (27) can be rewritten as

$$
\left\{\begin{array}{l}
\frac{d w_{1}}{d \tau}=w_{2}-\beta_{01}\left|w_{1}\right|^{\alpha_{1}} \operatorname{sign}\left(w_{1}\right) \\
\frac{d w_{2}}{d \tau}=w_{3}-\beta_{02}\left|w_{1}\right|^{\alpha_{2}} \operatorname{sign}\left(w_{1}\right) \\
\frac{d w_{3}}{d \tau}=-\beta_{03}\left|w_{1}\right|^{\alpha_{3}} \operatorname{sign}\left(w_{1}\right)-\eta
\end{array}\right.
$$

Neglecting $\eta$, Equation (29) can be replaced by

$$
\left\{\begin{array}{l}
\frac{d w_{1}}{d \tau}=w_{2}-\beta_{01}\left|w_{1}\right|^{\alpha_{1}} \operatorname{sign}\left(w_{1}\right) \\
\frac{d w_{2}}{d \tau}=w_{3}-\beta_{02}\left|w_{1}\right|^{\alpha_{2}} \operatorname{sign}\left(w_{1}\right) \\
\frac{d w_{3}}{d \tau}=-\beta_{03}\left|w_{1}\right|^{\alpha_{3}} \operatorname{sign}\left(w_{1}\right)
\end{array}\right.
$$

Definition 1 (see [35]). Let $f(x)=\left[f_{1}(x), \cdots, f_{n}(x)\right]^{T}: \mathrm{R}^{n} \rightarrow$ $\mathrm{R}^{n}$ as a vector field. If $\forall \varepsilon>0$, then $\left(r_{1}, \cdots, r_{n}\right) \in \mathrm{R}^{n}$ exists, where $r_{i}>0, i=1,2, \cdots, n$, and makes

$$
f_{i}\left(\varepsilon^{r_{1}} x_{1}, \cdots, \varepsilon^{r_{n}} x_{n}\right)=\varepsilon^{k+r_{i}} f_{i}(x), \quad i=1,2, \cdots, n,
$$

where $k>-\min \left\{r_{i}, i=1,2, \cdots, n\right\}$, and then the vector field $f(x)$ is homogeneous of degree $k$ with dilation $r=$ $\left(r_{1}, \cdots, r_{n}\right)$.

According to Definition 1, let $f_{w}$ stand for the vector function of system (29). When $r_{1}=1, r_{2}=(\alpha+2) / 3$, and $r_{3}=(2 \alpha+1) / 3$, there exists 


$$
f_{w}\left(c^{r_{1}} w_{1}, c^{r_{2}} w_{2}, \varepsilon^{r_{3}} x_{3}\right)=\left(\begin{array}{l}
c^{r_{2}} w_{2}-\beta_{01} c^{r_{1}}\left|w_{1}\right|^{\alpha_{1}} \operatorname{sign}\left(w_{1}\right) \\
c^{r_{3}} w_{3}-\beta_{02} c^{r_{1}}\left|w_{1}\right|^{\alpha_{2}} \operatorname{sign}\left(w_{1}\right) \\
-\beta_{03} c^{r_{1}}\left|w_{1}\right|^{\alpha_{3}} \operatorname{sign}\left(w_{1}\right)
\end{array}\right)=\left(\begin{array}{ccc}
c^{r_{1}+m} & 0 & 0 \\
0 & c^{r_{2}+m} & 0 \\
0 & 0 & c^{r_{3}+m}
\end{array}\right)\left(\begin{array}{l}
w_{2}-\beta_{01}\left|w_{1}\right|^{\alpha_{1}} \operatorname{sign}\left(w_{1}\right) \\
w_{3}-\beta_{02}\left|w_{1}\right|^{\alpha_{2}} \operatorname{sign}\left(w_{1}\right) \\
-\beta_{03}\left|w_{1}\right|^{\alpha_{3}} \operatorname{sign}\left(w_{1}\right)
\end{array}\right)
$$

where $m=(\alpha-1) / 3<0$, which is also homogeneous with vector function $f_{w}$ with dilation $\left(r_{1}, r_{2}, r_{3}\right)$.

Lemma 1 (see [36]). Suppose vector field $f(x)$ is homogeneous with degree $m$ with respect to $v$. Then, the origin is a finite-time-stable equilibrium under $f(x)$ if and only if the origin is an asymptotically stable equilibrium under $f(x)$ and $m<0$.

Lemma 2 (see [36]). For any continuous function $g(t)$ and $|g(t)|<\delta_{0}$, and considering the following disturbed system

$$
\dot{x}=f(x, t)+g(t) .
$$

If there is a $C^{1}$ smooth positive definite function $V(x)$, and for any $\beta_{1}>0$ and $\beta_{2} \in(0,0.5)$, which satisfies

$$
\dot{V}(x)+\beta_{1} V^{\beta_{2}}(x) \leq 0 .
$$

So there exists $L_{0}>0, \gamma_{0} \in(0,0.5)$, and a finite adjustment time $T_{\text {reach, }}$ and then the solution of system (33) satisfies

$$
\|x\| \leq L_{0} \delta_{0}^{\sigma_{0}},
$$

where $\sigma_{0}=\left(1-\gamma_{0}\right) / \gamma_{0}$.

In addition, according to the Routh stability criterion, $s^{3}+k_{3} s^{2}+k_{2} s+k_{1}$ is the Hurwitz polynomial only when $k_{3}>0, k_{1}>0$, and $k_{2}>k_{1} / k_{3}$. The origin of system (30) is the equilibrium point of asymptotic stability because of the linear correlation between the vector field $f_{w}$ and polynomial $s^{3}+k_{3} s^{2}+k_{2} s+k_{1}$. According to Lemma 1, the origin of system (30) is finite-time stable. Assuming that the upper limit of $|w(t)|$ is $w_{0}$, then $|\eta(\tau)| \leq w_{0} \varepsilon^{3}$. Next, by using Lemma 2 , in which there is a positive number $\mu$, this makes the solution of system (29) satisfy $|w(\tau)| \leq \mu$ $\left(w_{0} \varepsilon^{3}\right)^{\sigma}$ in finite time. Finally, according to Equation (28), it can be observed that the estimation errors are able to meet $\left|e_{i}\right| \leq\|w(\tau)\| \varepsilon^{-i+1}=L \varepsilon^{3 \sigma-i+1}$, where $L=\mu w_{0}^{\sigma}$, thus completing the stability proof.

Furthermore, to weaken the high-frequency chattering phenomenon in the simulation, the switching functions $\left|e_{1}\right|^{\alpha}$ sign $\left(e_{1}\right)$ in Equation (25) can be replaced by

$$
\operatorname{fal}\left(e_{1}, \alpha_{i}, \delta\right)= \begin{cases}\frac{e_{1}}{\delta^{\alpha_{i}-1}}, & \left|e_{1}\right| \leq \delta \\ \left|e_{1}\right|^{\alpha_{i}} \operatorname{sign}\left(e_{1}\right), & \left|e_{1}\right|>\delta\end{cases}
$$

where $\delta$ is a small positive constant determining the interval length of the linear segment.

3.2. On-Line Compensation Scheme. As shown in Figure 9, the angular acceleration induced by the different disturbance torque is estimated. Then, the additional LOS rate caused by the disturbance angular acceleration is also obtained on the basis of the DRR model of the seeker. Therefore, the LOS rate outputs of the seeker based on the $\mathrm{C}$ point and $\mathrm{S}$ point are compensated for by the additional LOS rate. The compensation scheme can be expressed as

$$
\begin{aligned}
G_{\widehat{q}_{D}}^{C}(s) & =\frac{K_{1}}{s^{2}+\left(K_{2} k_{g} / J\right) s+K_{1} K_{2} / J}, \\
G_{\overparen{\tilde{q}}_{D}}^{S}(s) & =\frac{-k_{g} s}{s^{2}+\left(K_{2} k_{g} / J\right) s+K_{1} K_{2} / J} .
\end{aligned}
$$

Analysis of the above compensation process shows that the scheme does not need to pay more attention to the specific form of the disturbance torque model but depends on the accurate dynamic model of the platform seeker. The above compensation scheme is different from that proposed in reference [16], where the linear filtering algorithm is adopted and errors exist in its deduction process.

\section{Simulations and Results}

In this section, numerical simulations are conducted to validate the effectiveness of the proposed compensation scheme. The DRR compensation effect is analyzed under different disturbance models. Secondly, the proposed DRR compensation scheme is applied to the missile guidance flight process, and its impact on miss distance is also analyzed.

4.1. Analysis of DRR Compensation Effect. The desired LOS rate which is $\dot{q}_{t}=0.5^{\circ} / \mathrm{s}$ and the disturbance angular acceleration are selected as the sinusoidal signal and colored noise signal, respectively, as shown in Figure 10. The proposed compensation scheme is executed to eliminate the effect of disturbance, and the observer gains are selected as $\beta_{01}=10, \beta_{02}=500, \beta_{03}=4680, \alpha=0.25$, and $\varepsilon=0.8$. The LOS rate outputs from the $C$ point and the $S$ point while considering the DRR compensation are shown in Figures 11 and 12. 




(a) Sinusoidal signal

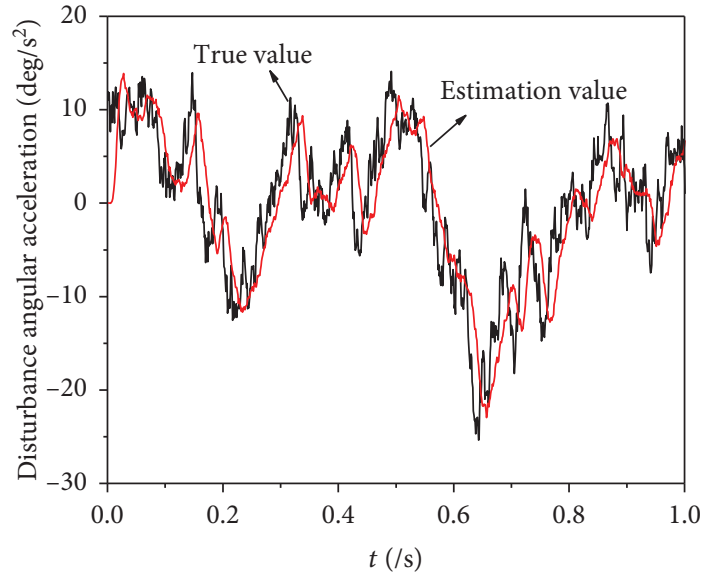

(b) Color noise signal

Figure 10: Disturbance angular acceleration.

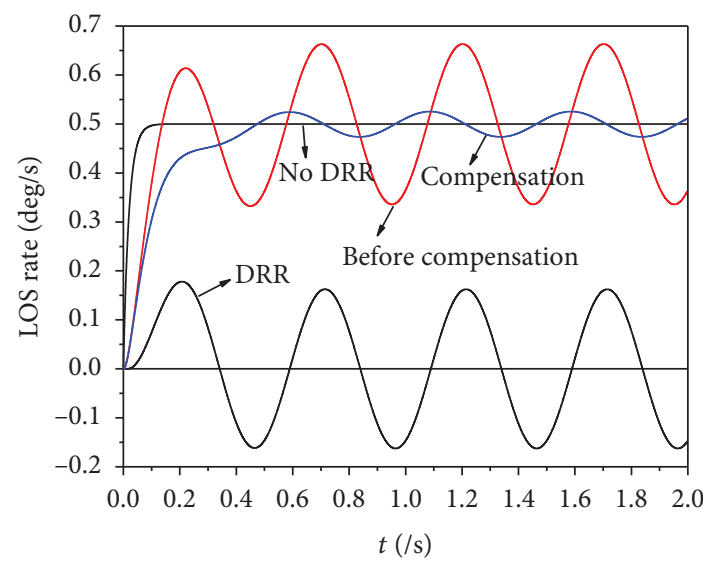

(a) C point output with sinusoidal disturbance

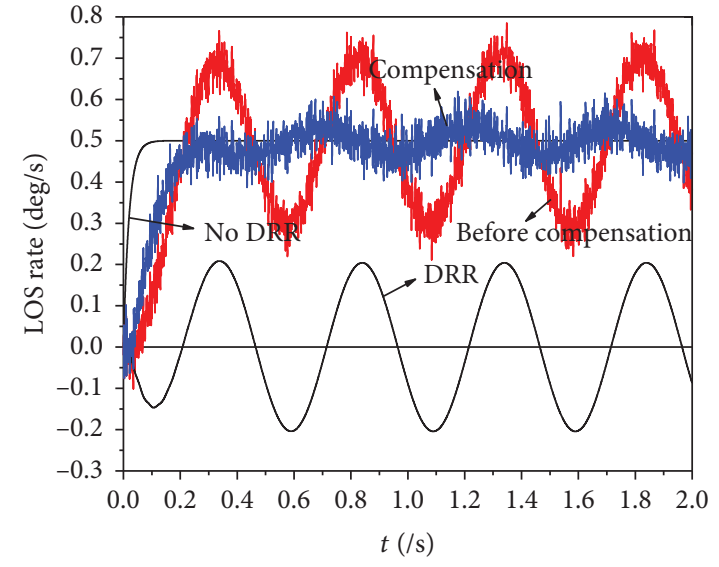

(b) $\mathrm{S}$ point output with sinusoidal disturbance

FIGURE 11: LOS rate outputs under sinusoidal disturbance.

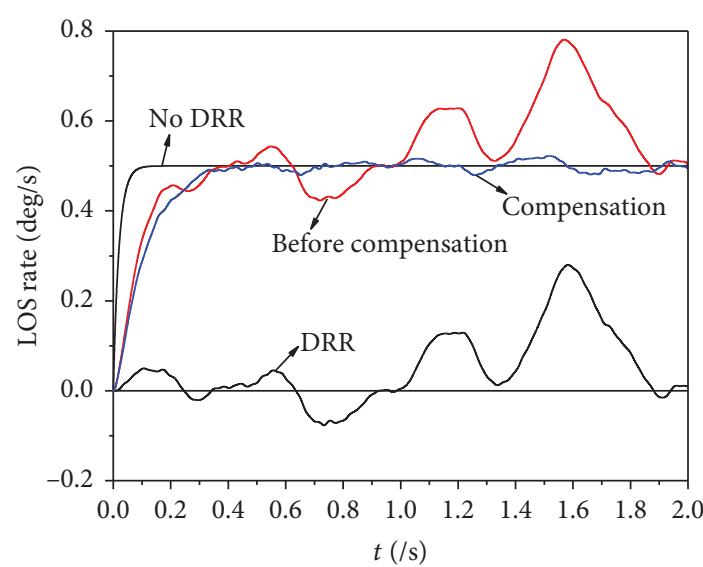

(a) C point output with color noise

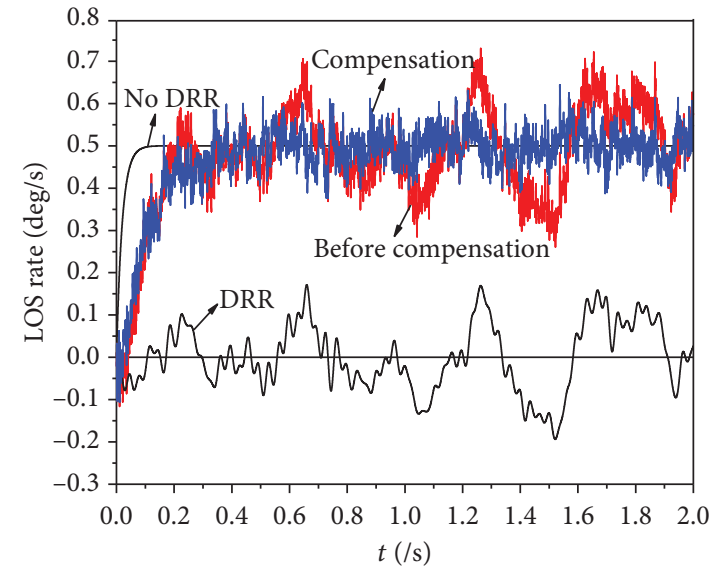

(b) $\mathrm{S}$ point output with color noise

FIgURE 12: LOS rate outputs under colored noise. 


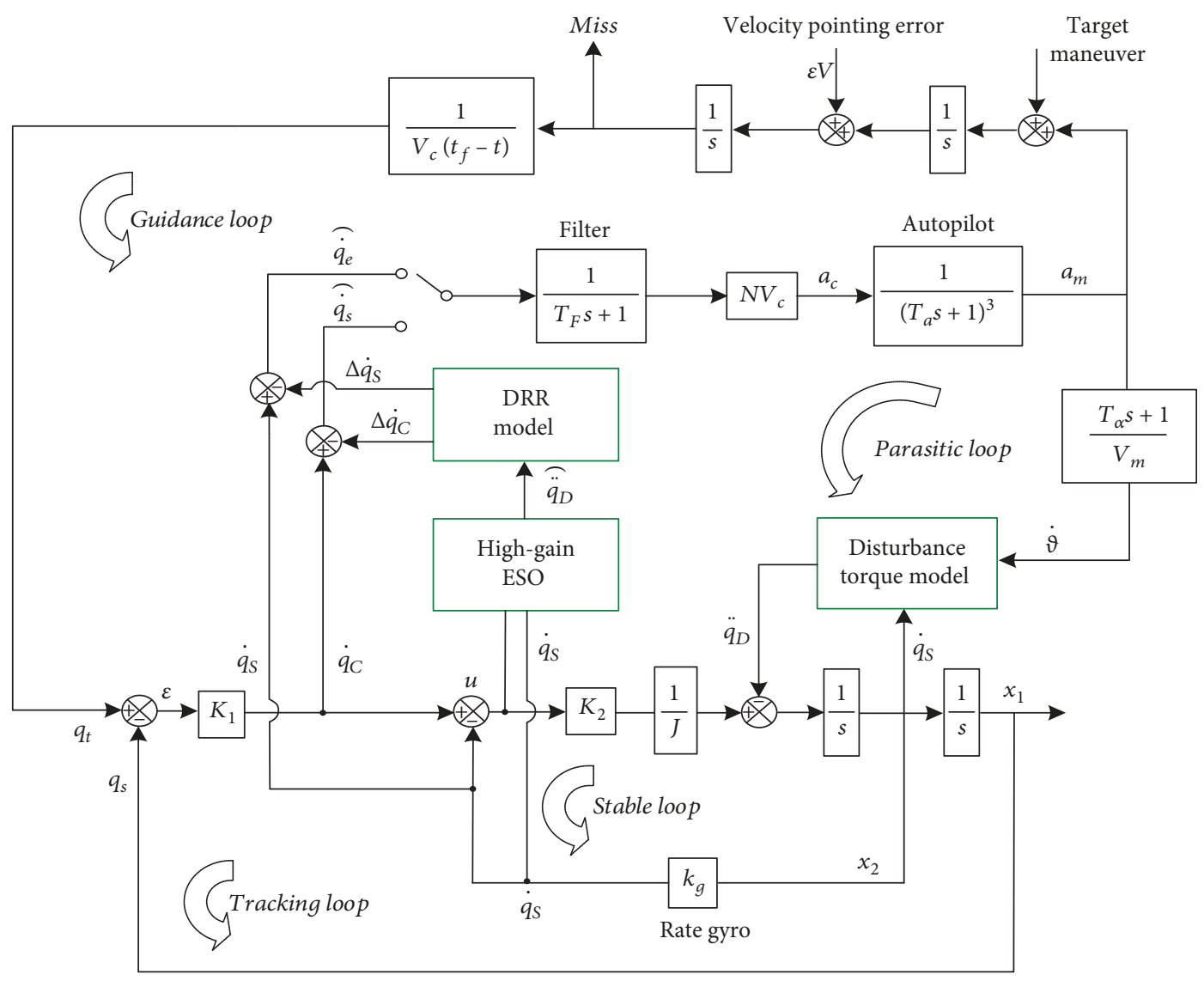

Figure 13: Block diagram of the missile guidance and control system.

TABle 3: Typical flight parameters of missile.

\begin{tabular}{lccc}
\hline Parameter & Value & Parameter & Value \\
\hline Navigation ratio & $N=4$ & Missile velocity & $V_{m}=450 \mathrm{~m} / \mathrm{s}$ \\
Closing velocity & $V_{c}=300 \mathrm{~m} / \mathrm{s}$ & Rate gyro gain & $k_{g}=1.0$ \\
Autopilot time constant & $T_{a}=0.1 \mathrm{~s}$ & Filtering time constant & $T_{F}=0.1 \mathrm{~s}$ \\
Guidance time constant & $T_{g}=0.5 \mathrm{~s}$ & Angle-of-attack time constant & $T_{\alpha}=1.0 \mathrm{~s}$ \\
\hline
\end{tabular}

In Figures 11(a) and 11(b), the estimated effects of the C point and $S$ point are compared under sinusoidal disturbance angular acceleration. Findings show that the LOS rate output from the $\mathrm{C}$ point is very smooth with less noise than the $\mathrm{S}$ point due to the effect of angular rate gyro noise. Moreover, the seeker DRR shows a sinusoidal trend and can be effectively suppressed by adopting the compensation scheme. However, the basic characteristics are consistent with that of the sinusoidal disturbance when the colored noise signal is used as the disturbance angular acceleration, as shown in Figure 12. Therefore, whatever angular acceleration is used as the disturbance signal, the proposed DRR compensation scheme can be used to estimate the disturbance moment signal and enhance the extraction accuracy of the LOS rate.
4.2. Influence of DRR Compensation on Miss Distance. In order to analyze the influence of the seeker DRR compensation scheme on the terminal miss distance of a missile, the block diagram of the missile guidance and control system is established, as shown in Figure 13. The decoupled LOS rate is transmitted to the proportional navigation law after firstorder filtering and then generating the required overload command signal. The guidance command is used as the input of a third-order autopilot model to generate the available overload for achieving the flight control of the missile. In addition, in Figure 13, errors mainly include target maneuvering and initial pointing error, and the initial pointing error is selected as the source of the whole simulation error.

The typical flight parameters of a missile are listed in Table 3, and the initial velocity pointing error angle and 


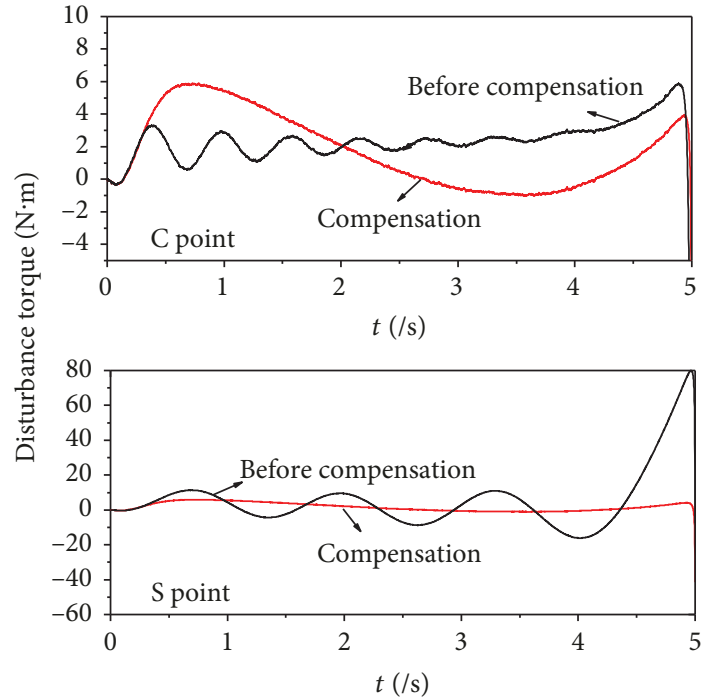

(a) Disturbance torque

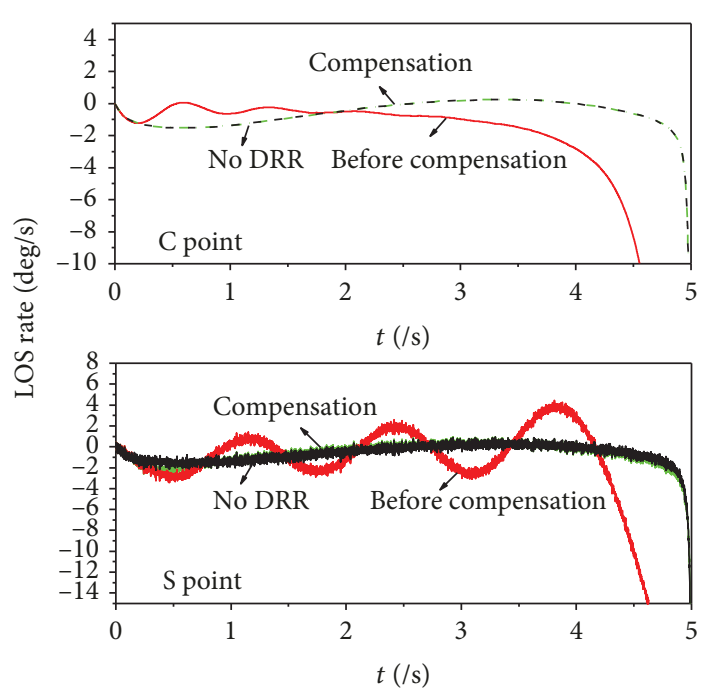

(b) LOS rate

FIgURe 14: Damping torque.

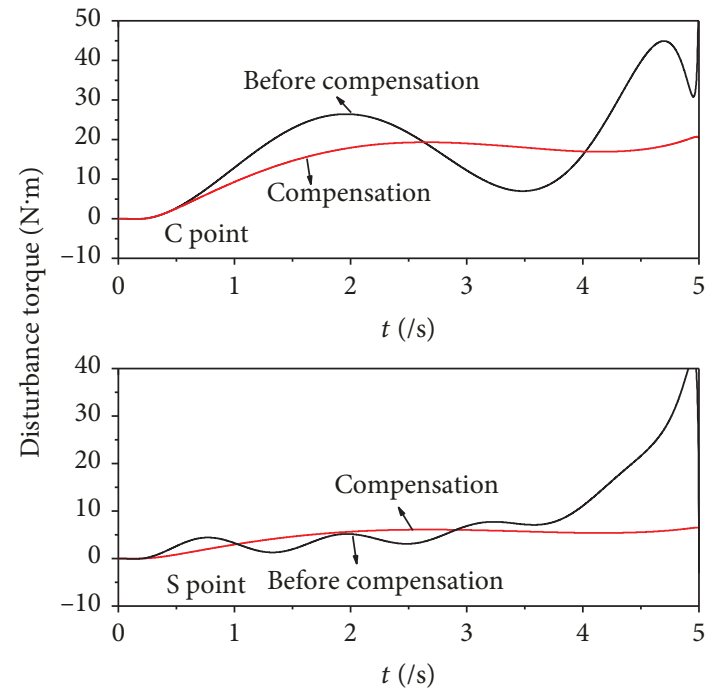

(a) Disturbance torque

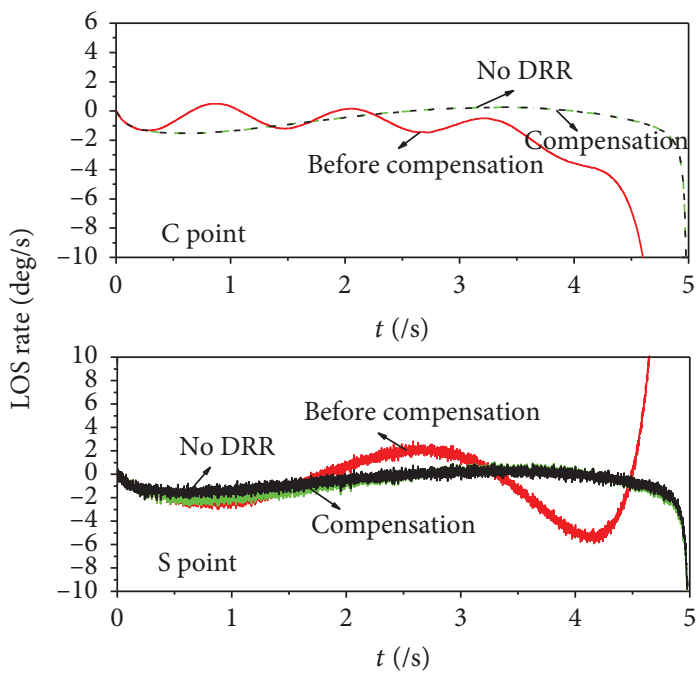

(b) LOS rate

Figure 15: Spring torque.

trajectory simulation time are chosen as $\varepsilon=10^{\circ}$ and $t_{f}=5 \mathrm{~s}$, respectively. Considering the influence of the seeker DRRPL caused by the damping moment and spring moment, the related simulation results are obtained and compared based on the proposed DRR compensation scheme, as shown in Figures 14-16.

The LOS rate outputs from the $\mathrm{C}$ point and S point are given in Figure 14 under damping torque. In Figure 14(a), the disturbance torque curves become smoother after being compensated, which indicates that the effect on the guidance and control system is less. However, in Figure 14(b), it can be observed that the LOS rate outputs using the compensation scheme keep consistent with no DRR situation. In all, the simulation results show that the proposed DRR compensa- tion scheme can weaken the effect of the damping torque and improve the stability of the missile guidance and control system significantly. The same conclusions can be obtained when the spring torque is the disturbance source, as shown in Figure 15.

The missile flight trajectory curves while considering the influences of the initial velocity pointing error and seeker DRR parasitical loop are shown in Figure 16. When the seeker disturbance torque is the damping torque, the results show that the trajectory curve from the $S$ point for guidance signal extraction appears with a certain diverge, and the terminal miss distance is relatively large. However for the $\mathrm{C}$ point, the trajectory curve appears to have a relatively large deviation and the miss distance is large. After adopting the 


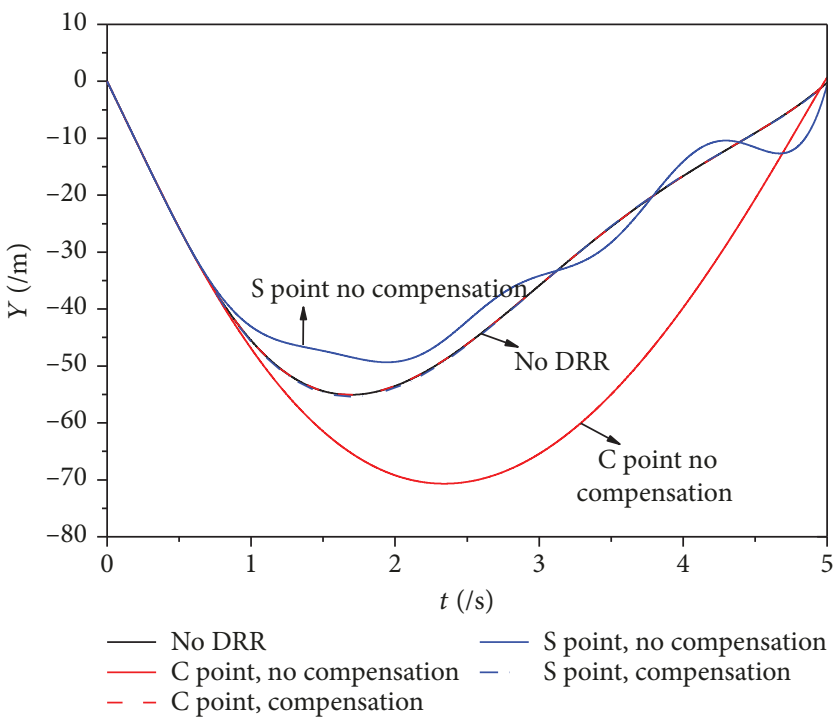

(a) Damping torque

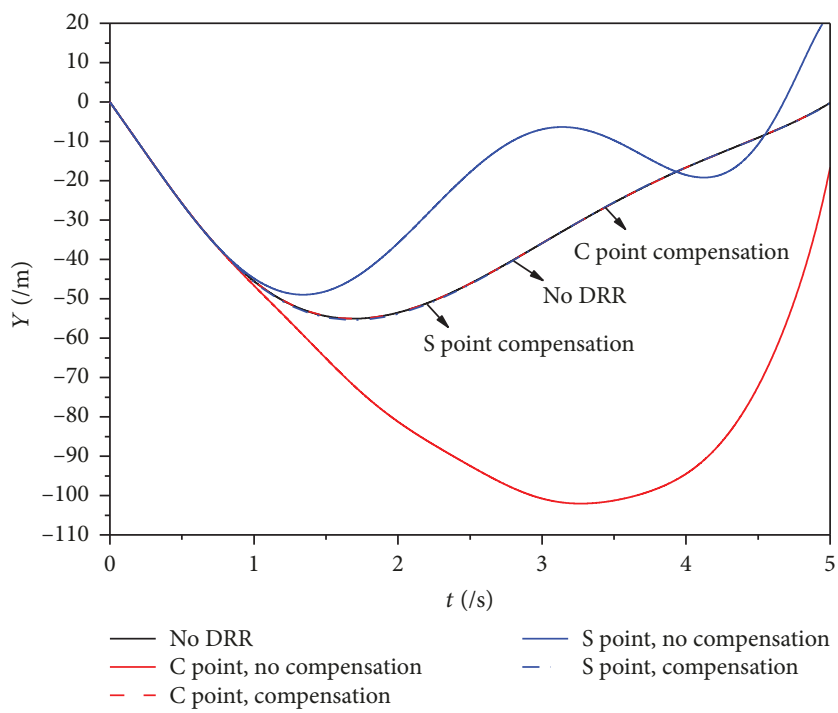

(b) Spring torque

FIGURE 16: Trajectory curves.

DRR compensation scheme, the trajectory curves of the guidance signal extracted from the $\mathrm{C}$ point and the $\mathrm{S}$ point are both smoother and the miss distances become smaller, which are similar to the situation without considering the DRR effect. In Figure 16(b), the same conclusions can be obtained when considering the spring torque disturbance.

In conclusion, all the simulation results above confirm that the seeker DRR compensation scheme based on the high-gain ESO can effectively suppress the negative impact of the DRRPL on the missile terminal flight. The scheme can be compared with reference [16], which proposed a linear filtering algorithm to estimate the damping torque and spring torque and then compensate the LOS rate outputs. Errors about the deduction process exist in reference [16], and related simulation results are given (see the Appendix). Comparing the simulation results of these two papers, the effect of the seeker DRR on both the missile guidance and the control system can be suppressed effectively, but our method has better robustness and is simpler to implement.

\section{Conclusions}

In this paper, the mathematical model of the platform seeker is built and the feasibility of the seeker structure concept is verified, which have laid the theoretical basis for the following research. The DRR model of the seeker is obtained considering different disturbance torques, and the amplitude and phase characteristics at different frequencies are also analyzed. Based on the reasonable assumptions, the DRRPL of the seeker is simplified and the stable region from different signal extraction points is obtained. The guidance and control system model of the missile is established considering the parasitic loop coupling, and a novel compensation scheme 


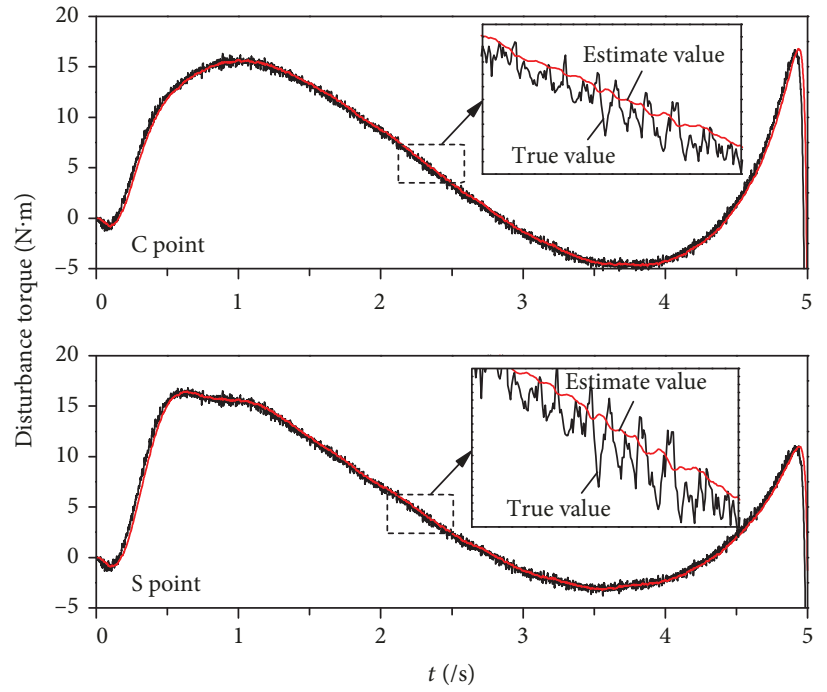

(a) Disturbance torque

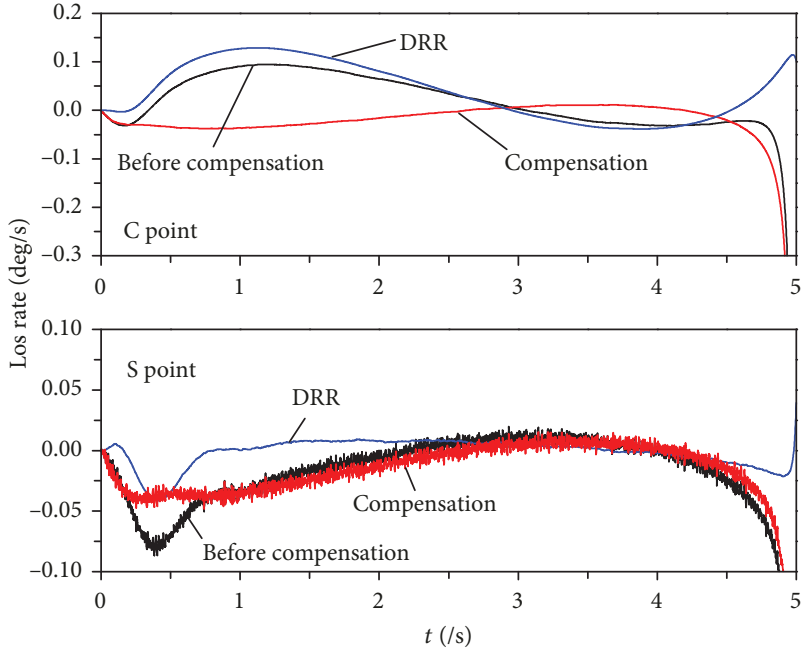

(b) Los rate

Figure 17: Damping torque.

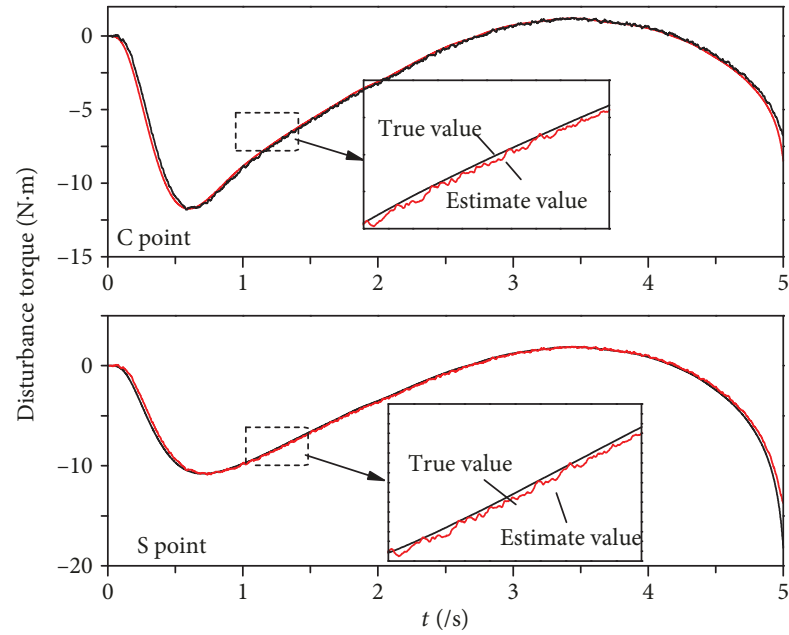

(a) Disturbance torque

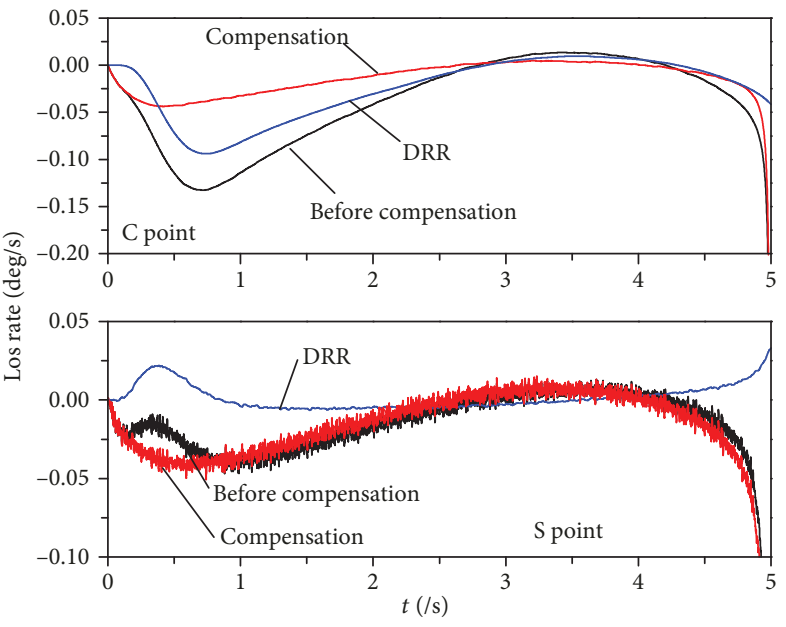

(b) Los rate

Figure 18: Spring torque.

is used to eliminate the DRR effect. Through numerical simulation validation, the following conclusions can be drawn:

(1) The output of the LOS rate from the S point lags slightly behind the $\mathrm{C}$ point. The outputs of the seeker are at a certain offset relative to the desired LOS rate under the influence of the detector zero deviation, while they chatter when the disturbance is detector noise.

(2) The stable region under the damping torque from the $\mathrm{C}$ point is wider than that from the $\mathrm{S}$ point, whereas the stable region under the spring torque from the $\mathrm{C}$ point is less than that from the $\mathrm{S}$ point

(3) The DRR effect of the seeker mainly manifests in the low and middle frequencies and can be well sup- pressed by the proposed compensation scheme. The compensation scheme can effectively improve missile guidance accuracy and reduce terminal miss distance

Our future work will focus on a more realistic seeker model and disturbance torque model and then verify the effectiveness of the proposed scheme through mathematical simulation and hardware-in-the-loop simulation.

\section{Appendix}

\section{Simulation Outputs and Related Conclusions in Reference [16]}

In reference [16], the disturbance torque curves and the LOS rate outputs are provided in Figures 17 and 18. 


\section{Data Availability}

The data used to support the findings of this study are included within the article.

\section{Conflicts of Interest}

The authors declare that there is no conflict of interests regarding the publication of this paper.

\section{Acknowledgments}

This work is supported in part by research grants from the Aviation Science Foundation, China (20150172001).

\section{References}

[1] H. R. Sonawane and S. P. Mahulikar, "Tactical air warfare: generic model for aircraft susceptibility to infrared guided missiles," Aerospace Science and Technology, vol. 15, no. 4, pp. 249-260, 2011.

[2] F. W. Nesline and P. Zarchan, "Radome induced miss distance in aerodynamically controlled homing missiles," in 17th Fluid Dynamics, Plasma Dynamics, and Lasers Conference, Snowmass, CO, USA, June 1984.

[3] S. Sadhu and T. K. Ghoshal, "Sight line rate estimation in missile seeker using disturbance observer-based technique," IEEE Transactions on Control Systems Technology, vol. 19, no. 2, pp. 449-454, 2011.

[4] V. Saini and H. B. Hablani, "Air-to-air tracking of a maneuvering target with gimbaled radar," Journal of Guidance, Control, and Dynamics, vol. 39, no. 2, pp. 262-274, 2016.

[5] C.-L. Lin and Y.-H. Hsiao, "Adaptive feedforward control for disturbance torque rejection in seeker stabilizing loop," IEEE Transactions on Control Systems Technology, vol. 9, no. 1, pp. 108-121, 2001.

[6] P. G. Savage, "Strapdown inertial navigation integration algorithm design part 1: attitude algorithms," Journal of Guidance, Control, and Dynamics, vol. 21, no. 1, pp. 19-28, 1998.

[7] W. W. Willman, "Effects of strapdown seeker scale-factor uncertainty on optimal guidance," Journal of Guidance, Control, and Dynamics, vol. 11, no. 3, pp. 199-206, 1988.

[8] S.-A. Jang, C.-K. Ryoo, K. Choi, and M.-J. Tahk, "Guidance algorithms for tactical missiles with strapdown seeker," in 2008 SICE Annual Conference, Tokyo, Japan, August 2008.

[9] Y. Y. Cui, Q. L. Xia, and Z. K. Qi, "Seeker platform disturbance rejection mathematical model," Journal of Projectiles, Rockets, Missiles and Guidance, vol. 82, no. 1, pp. 22-25, 2006.

[10] F. Li, Q. Xia, Z. Qi, and J. Sun, "Effect of parasitic loop on strap-down seeker and compensated with identification method," Systems Engineering and Electronics, vol. 8, p. 24, 2013.

[11] P. Garnell, Guided Weapon Control Systems, Second Revision by QiZaikang, Beijing Institute of Technology, 2004.

[12] Y. L. Du, Q. L. Xix, and Z. K. Qi, "Research on effect of seeker disturbance rejection rate with phase lag on stability of parasitical loop," Acta Armamentarii, vol. 32, no. 1, pp. 28-32, 2011.

[13] F. Li, Q. Xia, X. Cui, and Z. Qi, "Effect of seeker disturbance rejection rate parasitic loop on line of sight rate extraction," Journal of Astronautics, vol. 34, no. 8, pp. 1072-1077, 2013.
[14] T. Song, D. F. Lin, and J. Wang, "Influence of seeker disturbance rejection rate on missile guidance system," Journal of Harbin Engineering University, vol. 34, no. 10, pp. 12341241, 2013.

[15] X. Du and Q. Xia, "The research of guidance performance of the phased array seeker with platform for air-to-air missile," Optik, vol. 127, no. 22, pp. 10322-10334, 2016.

[16] S. Liu, X. Du, and Q. Xia, "An on-line compensation method for the disturbance rejection rate of seekers," Optik, vol. 157, pp. 1306-1318, 2018.

[17] A. Bhattacharyya and R. N. Bhattacharjee, "Performance analysis of nominal scheme and decoupling loop scheme for RF seeker," in AIAA Guidance, Navigation, and Control Conference and Exhibit, Monterey, CA, USA, August 2002.

[18] J. Xu, J. Wang, T. Song, and K. R. Hu, “A disturbance observerbased inhibition method for disturbance rejection rate of seeker," Acta Armamentarii, vol. 35, no. 11, pp. 1790-1798, 2014.

[19] K. B. Lee and F. Blaabjerg, "Robust and stable disturbance observer of servo system for low-speed operation," IEEE Transactions on Industry Applications, vol. 43, no. 3, pp. 627-635, 2007.

[20] B. K. Kim and W. K. Chung, "Advanced disturbance observer design for mechanical positioning systems," IEEE Transactions on Industrial Electronics, vol. 50, no. 6, pp. 1207-1216, 2003.

[21] D. Chwa, J. Y. Choi, and S. G. Anavatti, "Observer-based adaptive guidance law considering target uncertainties and control loop dynamics," IEEE Transactions on Control Systems Technology, vol. 14, no. 1, pp. 112-123, 2006.

[22] V.-P. Vu and W. J. Wang, "State/disturbance observer and controller synthesis for the T-S fuzzy system with an enlarged class of disturbances," IEEE Transactions on Fuzzy Systems, vol. 26, no. 6, pp. 3645-3659, 2018.

[23] Q. Li, J. Yuan, and B. Zhang, "Extended state observer based output control for spacecraft rendezvous and docking with actuator saturation," ISA Transactions, vol. 88, pp. 37-49, 2019.

[24] L. Zhao, B. Zhang, H. Yang, and Y. Wang, "Finite-time tracking control for pneumatic servo system via extended state observer," IET Control Theory and Applications, vol. 11, no. 16, pp. 2808-2816, 2017.

[25] X. Shao, J. Liu, H. Cao, C. Shen, and H. Wang, "Robust dynamic surface trajectory tracking control for a quadrotor UAV via extended state observer," International Journal of Robust and Nonlinear Control, vol. 28, no. 7, pp. 2700-2719, 2018.

[26] H. Gouta, S. Hadj Saïd, A. Turki, and F. M'Sahli, "Experimental sensorless control for a coupled two-tank system using high gain adaptive observer and nonlinear generalized predictive strategy," ISA Transactions, vol. 87, pp. 187-199, 2019.

[27] P. Wangze, Introduction to Radar Seeker, Electronic Industry Press, Beijing, 2010.

[28] C.-Y. Zheng, H.-G. Zhang, X.-W. Feng, and J.-K. Gao, "ADRC of airborne electro-optical stabilized platform," Electronies Optics \& Control, vol. 24, no. 2, pp. 51-55, 2017.

[29] S. Cong, G. Sun, K. Deng, W. Shang, and H. Shen, "Active disturbance rejection and filter control of gyro-stabilized platform," Optics and Precision Engineering, vol. 24, no. 1, pp. 169-177, 2016. 
[30] B. Yang and Y. Zhao, "Autopilot design method for the blended missile based on model predictive control," International Journal of Aerospace Engineering, vol. 2015, 13 pages, 2015.

[31] S. Liu, T. Lu, T. Shang, and Q. Xia, "Dynamic modeling and coupling characteristic analysis of two-axis rate gyro seeker," International Journal of Aerospace Engineering, vol. 2018, Article ID 8513684, 14 pages, 2018.

[32] J. Liu, Y. Gao, X. Su, M. Wack, and L. Wu, "Disturbanceobserver-based control for air management of PEM fuel cell systems via sliding mode technique," IEEE Transactions on Control Systems Technology, vol. 27, no. 3, pp. 1129-1138, 2019.

[33] Y. Yin, J. Liu, J. A. Sanchez et al., "Observer-based adaptive sliding mode control of NPC converters: an RBF neural network approach," IEEE Transactions on Power Electronics, vol. 34, no. 4, pp. 3831-3841, 2019.

[34] J. Liu, Y. Yin, W. Luo, S. Vazquez, L. G. Franquelo, and L. Wu, "Sliding mode control of a three-phase AC/DC voltage source converter under unknown load conditions: industry applications," IEEE Transactions on Systems, Man, and Cybernetics: Systems, vol. 48, no. 10, pp. 1771-1780, 2018.

[35] Y. Hong, J. Huang, and Y. Xu, "On an output feedback finitetime stabilisation problem," in Proceedings of the 38th IEEE Conference on Decision and Control (Cat. No.99CH36304), Phoenix, AZ, USA, December 1999.

[36] S. P. Bhat and D. S. Bernstein, "Geometric homogeneity with applications to finite-time stability," Mathematics of Control, Signals, and Systems, vol. 17, no. 2, pp. 101-127, 2005. 




\section{Enfincering}


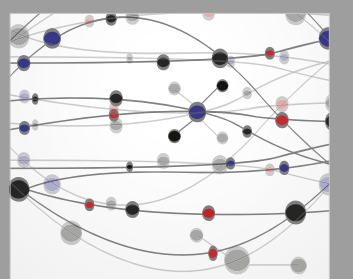

\section{Rotating \\ Machinery}

The Scientific World Journal

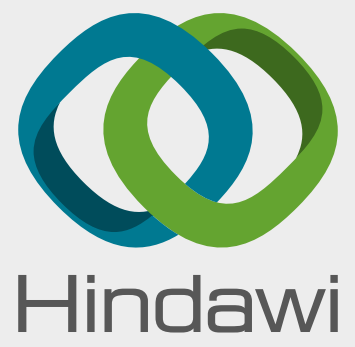

Submit your manuscripts at

www.hindawi.com
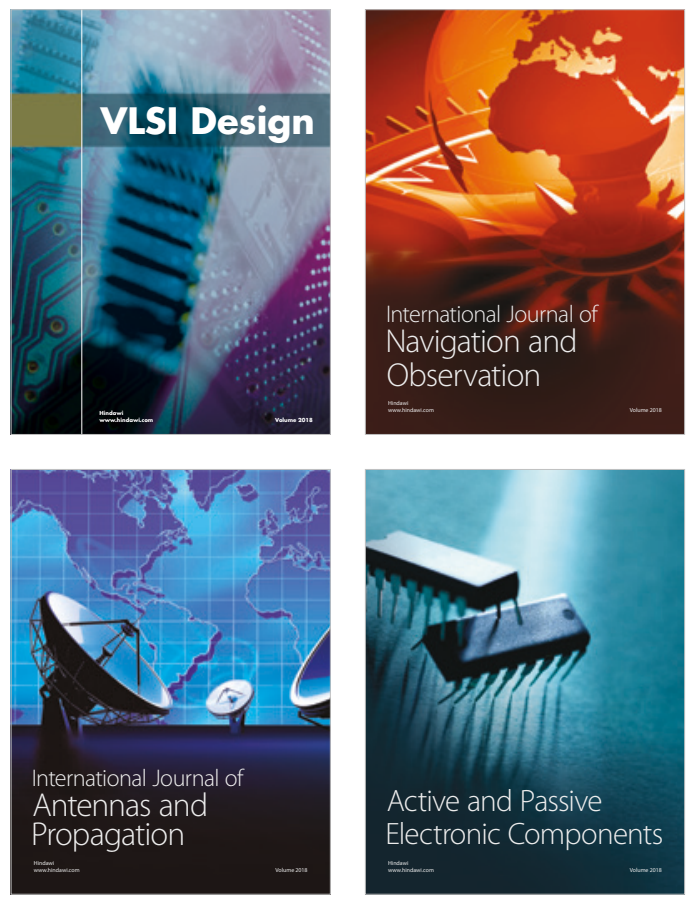
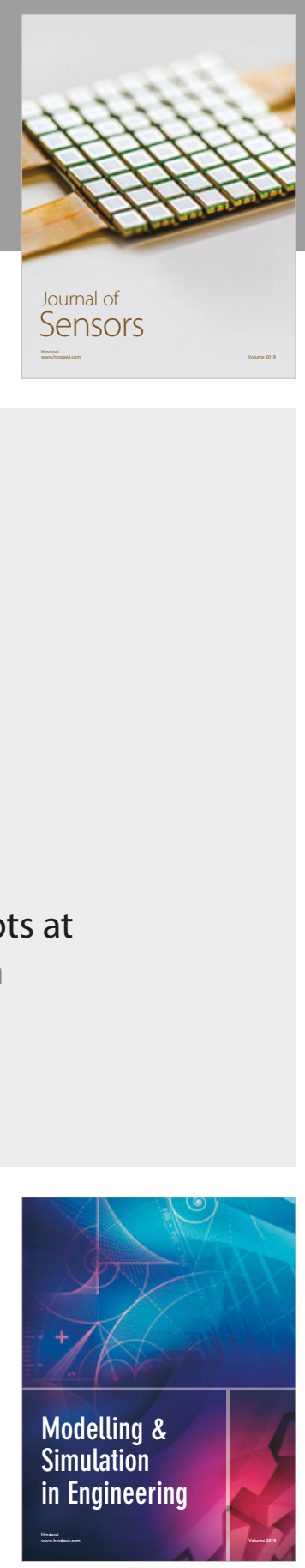

\section{Advances \\ Multimedia}
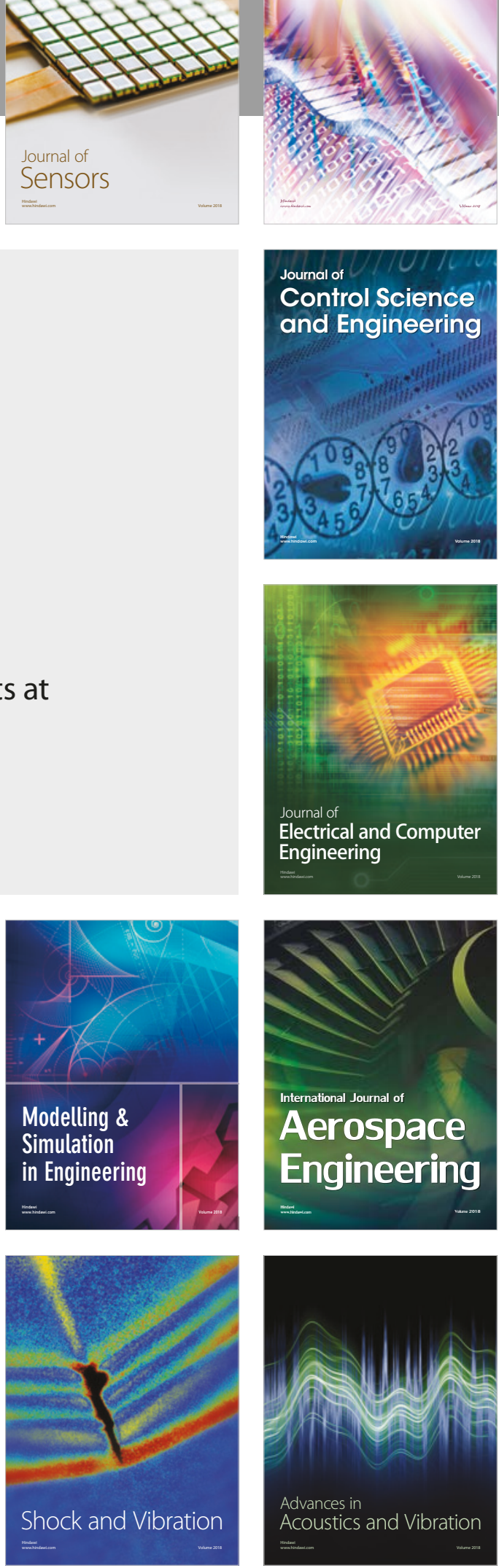